\title{
Effects of Pulse Phase Duration and Location of Stimulation Within the Inferior Colliculus on Auditory Cortical Evoked Potentials in a Guinea Pig Model
}

\author{
Anke Neuheiser $^{1,2}$, Minoo Lenarz ${ }^{1}$, Guenter Reuter ${ }^{1,4}$, Roger Calixto ${ }^{1}$, Ingo Nolte ${ }^{2}$, \\ Thomas Lenarz ${ }^{1}$, AND Hubert H. Lim ${ }^{1,3}$ \\ ${ }^{1}$ Otorhinolaryngology Department, Hannover Medical University, 30625 Hannover, Germany \\ ${ }^{2}$ Small Animal Clinic, University of Veterinary Medicine Hannover, 30173 Hannover, Germany \\ ${ }^{3}$ Biomedical Engineering Department, University of Minnesota, 55455 Minneapolis, MN, USA \\ ${ }^{4}$ Medizinische Hochschule Hannover, Carl-Neuberg-Str. 1, OE 6500, 30625 Hannover, Germany
}

Received: 27 April 2009; Accepted: 23 July 2010; Online publication: 18 August 2010

\begin{abstract}
The auditory midbrain implant (AMI), which consists of a single shank array designed for stimulation within the central nucleus of the inferior colliculus (ICC), has been developed for deaf patients who cannot benefit from a cochlear implant. Currently, performance levels in clinical trials for the AMI are far from those achieved by the cochlear implant and vary dramatically across patients, in part due to stimulation location effects. As an initial step towards improving the AMI, we investigated how stimulation of different regions along the isofrequency domain of the ICC as well as varying pulse phase durations and levels affected auditory cortical activity in anesthetized guinea pigs. This study was motivated by the need to determine in which region to implant the single shank array within a threedimensional ICC structure and what stimulus parameters to use in patients. Our findings indicate that complex and unfavorable cortical activation properties are elicited by stimulation of caudal-dorsal ICC regions with the AMI array. Our results also confirm the existence of different functional regions along the isofrequency domain of the ICC (i.e., a caudal-
\end{abstract}

Anke Neuheiser and Minoo Lenarz contributed equally as first authors.

Correspondence to: Guenter Reuter · Medizinische Hochschule Hannover · Carl-Neuberg-Str. 1, OE 6500, 30625 Hannover, Germany. Telephone: +49-511-5324932; fax: +49-511-5328930; email: Reuter.Guenter@MH-Hannover.de dorsal and a rostral-ventral region), which has been traditionally unclassified. Based on our study as well as previous animal and human AMI findings, we may need to deliver more complex stimuli than currently used in the AMI patients to effectively activate the caudal ICC or ensure that the single shank AMI is only implanted into a rostral-ventral ICC region in future patients.

Keywords: auditory midbrain implant, auditory brainstem implant, cochlear implant, deep brain stimulation, auditory cortex, auditory thalamus

\section{INTRODUCTION}

The auditory midbrain implant (AMI) is a new neural prosthesis designed for electrical stimulation of the inferior colliculus (IC) for hearing restoration (Reuter et al. 2004; Lenarz et al. 2006b; Lim et al. 2009). It was developed as an alternative to the auditory brainstem implant (ABI), which consists of a planar array placed onto the surface of the cochlear nucleus in neurofibromatosis type II (NF2) patients undergoing acoustic neuroma removal surgery (Brackmann et al. 1993; Schwartz et al. 2008). The surgery requires exposure of the brainstem for tumor removal and usually leads to complete deafness in the patient, thus justifying implantation of the ABI. The poor performance in ABI patients (i.e., limited or no open-set speech perception) has been attributed to tumor-related damage at the level of the brainstem (Colletti and 
Shannon 2005; Colletti et al. 2009). Thus, the IC, which is not directly damaged by the tumor and has shown to be surgically accessible during tumor removal surgery (Samii et al. 2007), provides a potential alternative to the cochlear nucleus as a target for a hearing prosthesis.

The AMI is currently in clinical trials in which a total of five NF2 patients have been implanted with a single shank array (Fig. 1A) into the auditory midbrain. Although the intended region was the central nucleus of the IC (ICC), the main ascending auditory midbrain region, placement varied dramatically across patients. Initial results have been published for the first three patients (Lim et al. 2007, 2008b). Overall, the patients obtain improvements in lip-reading capabilities and environmental awareness. However, similar to the performance in ABI patients, their open-set speech perception is limited. One ongoing hypothesis is that none of the AMI patients are implanted within an optimal region. Only one patient was implanted into the target region, the ICC, and exhibits higher performance levels than those implanted outside the ICC, such as within the dorsal cortex or brachium of the inferior colliculus. However, even for the ICC-implanted patient, activation thresholds were higher than expected for direct neural activation. Based on animal studies, there appears to exist at least two spatially distinct functional regions organized along the isofrequency domain of the ICC (i.e., a caudal-dorsal and a rostral-ventral region) that transmit sound information in different ways to the auditory cortex ( Lim and Anderson 2007a; Lim et al. 2008a). In particular, the caudal-dorsal region exhibits dramatically higher stimulation thresholds and discriminable level steps, reduced synchronized and temporally diffuse activation, and possibly less focused tonotopic activation than the rostral-ventral region. These neural features may correspond to degraded level, temporal, and spectral coding, which all have shown to be important for speech perception (Shannon et al. 1995, 2004; Zeng and Galvin 1999; Loizou et al. 2000; Friesen et al. 2001; Rance et al. 2002). Thus, these animal results may explain the elevated thresholds and limited performance in the ICC-implanted patient whose array is actually positioned into a caudal-dorsal region (Lim et al. 2007). An optimal target in future patients may be the rostral-ventral region of the ICC.

The animal studies described above used "Michigan" silicon substrate multi-site probes for ICC stimulation with iridium sites that were $400 \mu \mathrm{m}^{2}$. The AMI array used in humans (Fig. 1A) consists of platinum rings that have a considerably larger surface area of $126,000 \mu \mathrm{m}^{2}$, which was constrained by the implant technology currently approved for human use. Therefore, it is important to assess if similar location effects observed in those previous studies also occur for stimulation with the human AMI array considering the significant difference in site area that can result in dissimilar activation patterns. Furthermore, those previous studies only used biphasic pulses with a phase duration (PD) of $200 \mu$ s and a narrow range of current levels due to the limited current injection capacity of the smaller iridium sites. As an initial step towards identifying appropriate regions and stimuli for effective ICC activation with the human AMI array, the focus of this study was to investigate how stimulation with varying PDs and levels across different locations along the isofrequency domain of the ICC affects neural activity within the primary auditory cortex (A1) of guinea pigs. The results are then compared to previous animal studies to assess if AMI stimulation can sufficiently activate higher auditory

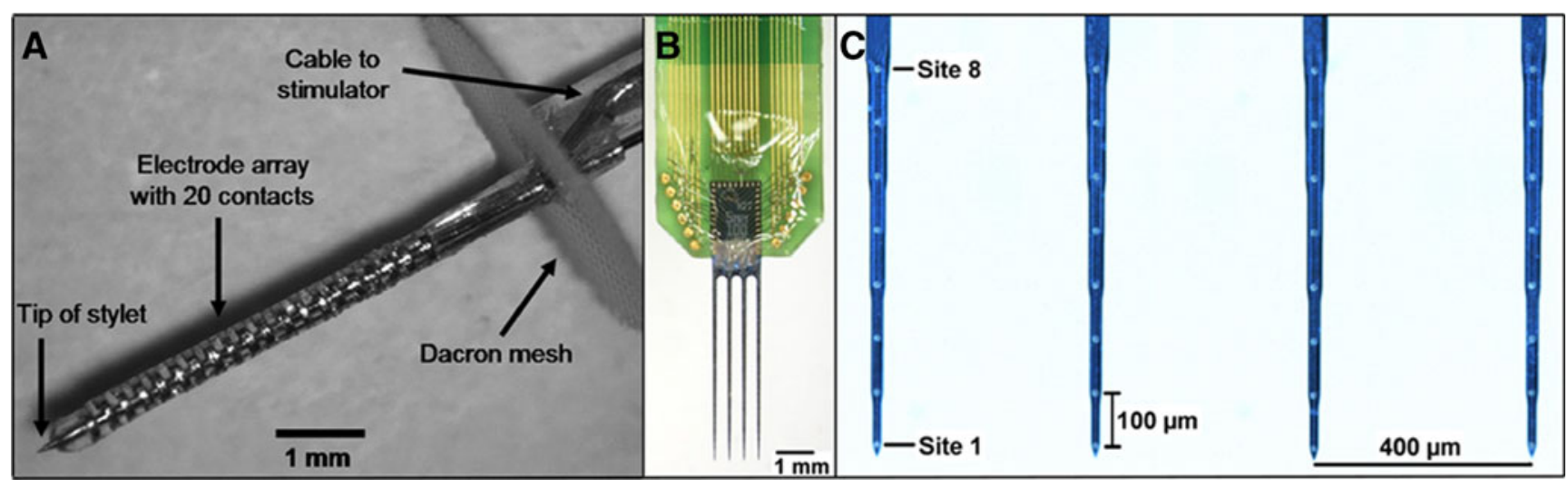

FIG. 1. A Image of the AMI array developed by Cochlear Ltd. (Lane Cove, Australia). The array is $6.2 \mathrm{~mm}$ long with a diameter of $0.4 \mathrm{~mm}$. It consists of 20 platinum ring electrodes linearly spaced at an interval of $200 \mu \mathrm{m}$. Each site has a thickness of $100 \mu \mathrm{m}$ (surface area of $126,000 \mu \mathrm{m}^{2}$ ). A stainless steel stylet is positioned through the axial center of the silicone carrier to enable insertion of the array into the brain and is intended for removal after placement for human implantation. Further details on the AMI electrode array are included in Lenarz et al. (2006b). B A multi-site silicon substrate "Michigan" probe consisting of four shanks magnified in $\mathbf{C}$ that were each separated by $400 \mu \mathrm{m}, 5 \mathrm{~mm}$ long and $15 \mu \mathrm{m}$ thick. Along each shank, eight iridium sites ( $400 \mu \mathrm{m}^{2}$ surface area) were linearly spaced at a distance of $100 \mu \mathrm{m}$. 
centers within neural stimulation safety limits to elicit intelligible speech perception.

\section{METHODS}

Basic surgical procedures and methods for neural recording and stimulation were similar to those presented in previous works (Lenarz et al. 2006a; Lim and Anderson 2006, 2007a). For this study, we electrically stimulated different regions along the isofrequency domain of the ICC using the human AMI single shank array (multiple penetrations aligned along the tonotopic axis) and recorded the corresponding neural activity within A1 that had a similar best frequency (BF) to the stimulated ICC sites using multi-site probes in anesthetized guinea pigs. Current source density analysis, acoustic-driven responses, and histological techniques were used to confirm that all sites were correctly positioned within the ICC and A1. We then assessed the effects of varying PDs and levels for single pulse stimulation within the ICC on neural activity within A1.

In this paper, we only present the results for the local field potentials recorded on our A1 sites. We have been unable to analyze the spike data due to issues associated with antidromic activation. Electrical stimulation of the ICC can antidromically activate neurons projecting from A1. This was demonstrated in a previous study ( $\mathrm{Lim}$ and Anderson 2007b) in which stimulation throughout the ICC antidromically activated neurons originating predominantly from layer $\mathrm{V}$ of $\mathrm{A} 1$. There were a few cases in which antidromic spikes were recorded from other layers. Generally, antidromic spikes could be identified and differentiated from orthodromic spikes based on latency jitter, spike shape, and effects to high pulse rates. In this study, we observed a greater extent of antidromic activity across multiple cortical layers in which overlapping spikes were frequently observed on a given recording site. The stimulation sites used in this study were considerably larger than those used in Lim and Anderson (2007b) $\left(126,000 \mu \mathrm{m}^{2}\right.$ versus $\left.400 \mu \mathrm{m}^{2}\right)$; thus, they activated a larger number of neurons projecting from A1 to the ICC. As a result, we have not yet been able to accurately isolate the orthodromically activated spikes from the antidromically activated spikes.

\section{Anesthesia and surgery}

All experiments were performed on young male pigmented guinea pigs (375-560 g; BFA; Charles River WIGA GmbH, Sulzfeld, Germany). The animals were initially anesthetized with an intramuscular injection of ketamine $(40 \mathrm{mg} / \mathrm{kg}$ ) and xylazine $(10 \mathrm{mg} / \mathrm{kg})$. Injections of ketamine and xylazine in a 4:1 mixture were administered periodically to main- tain an areflexive state. Atropine sulfate $(0.05 \mathrm{mg} / \mathrm{kg})$ was injected subcutaneously to reduce bronchial secretions. A warm water heating blanket controlled by a rectal temperature probe was used to keep the body temperature at $38 \pm 0.5^{\circ} \mathrm{C}$. The heart rate and blood oxygen level were continuously monitored throughout the experiment via pulsoximetry.

The animals were fixed into a stereotaxic frame (David Kopf Instruments, Tujunga, CA, USA). After doing a skin incision along the midline of the scalp to expose the right side of the skull, a craniotomy was performed to expose the brain from the caudal end of the occipital lobe to the pseudosylvian sulcus of the temporal lobe. The dura above the auditory cortex was removed and a multi-site silicon substrate Michigan probe (Fig. 1B, C; NeuroNexus Technologies, Ann Arbor, MI, USA) was inserted into A1 using a micromanipulator. The A1 probe consisted of four shanks, each separated by $400 \mu \mathrm{m}, 5 \mathrm{~mm}$ long and $15 \mu \mathrm{m}$ thick. Along each shank, eight iridium sites were linearly placed at a distance of $100 \mu \mathrm{m}$ (all distances center-tocenter) and each site had an area of $400 \mu \mathrm{m}^{2}$ and an impedance between 1 and $2 \mathrm{M} \Omega$ (at $1 \mathrm{kHz}$ ). The 32site A1 probe was inserted approximately perpendicular to the cortical surface aligning each shank along a BF column of A1 (Abeles and Goldstein 1970; Redies et al. 1989; Wallace et al. 2000). After appropriate placement of the A1 probe based on acousticdriven responses (see Methods: "Placement of probes"), we covered the exposed brain with agarose to reduce swelling, pulsations, and drying.

For AMI array placement, we removed the dura over the occipital cortex, which was then partially aspirated to provide direct visualization of the right IC surface (Bledsoe et al. 2003; Snyder et al. 2004). The AMI array (Fig. 1A) was inserted at a $45^{\circ}$ angle to the sagittal plane into the IC to align the array along the tonotopic axis of the ICC (Merzenich and Reid 1974; Schreiner and Langner 1997; Snyder et al. 2004; Lenarz et al. 2006a; Lim and Anderson 2006). The AMI sites had impedances of $5-20 \mathrm{k} \Omega$ (at $1 \mathrm{kHz}$ ). It was not necessary to remove the stylet as what occurs during human implantation since the array was not chronically implanted (Samii et al. 2007). During each experiment, one to four AMI array placements were made. For a separate series of experiments (eight animals), in which part of the data is presented in this paper, we simultaneously implanted two single shank arrays at a separation of $1.5 \mathrm{~mm}$ to ensure one shank was in a caudal-dorsal region and the other within a rostral-ventral region. The brain was covered with agarose after each array placement.

\section{Stimulation and recording setup}

All experiments were performed in a sound-attenuating and electrically shielded room and controlled by a 
computer interfaced with TDT hardware (TuckerDavis Technology, Alachua, FL, USA) using custom software written in Matlab (MathWorks, Natick, MA, USA). For acoustic stimulation, sound was presented via a speaker coupled to the left ear through a hollow ear bar. The speaker-ear bar system was calibrated using a 0.25-in. condenser microphone (ACO Pacific, Belmont, CA, USA) where the tip of the ear bar was inserted into a short plastic tube with the microphone inserted into the other end. The tube represented the ear canal.

To monitor the placement of both electrode arrays, we presented various levels of pure tones and broadband noise that were $50 \mathrm{~ms}$ in duration with 5 and $0.5 \mathrm{~ms}$ rise-fall ramp times, respectively, to elicit acoustic-driven activity in the contralateral ICC and A1. All neural signals were passed through analog DCblocking and anti-aliasing filters from $1.6 \mathrm{~Hz}$ to $7.5 \mathrm{kHz}$.

After placement of both probes, the AMI array was connected to an optically isolated current stimulator. The selected AMI sites were stimulated in monopolar configuration with the return through a wire into the neck muscles. The electrical stimuli consisted of single, biphasic, charge-balanced pulses (cathodicleading) from 3 to $794 \mu \mathrm{A}$ in 2-dB steps (relative to $1 \mu \mathrm{A}$ ). We used several PDs between 41 and 1,476 $\mu \mathrm{s}$ (i.e., 41, 82, 123, 205, 328, 410, 492, 656, 820, 984, $1,476 \mu \mathrm{s})$. Not all animals were presented with the same list of PDs. The PD of $41 \mu$ s was the shortest we could deliver with our stimulation setup. Each stimulus was presented 20 times at a rate of $2 / \mathrm{s}$ in a randomized sequence across all PDs and levels (including 20 spontaneous trials) to reduce adaptive effects. Neural data were recorded on all $32 \mathrm{~A} 1$ sites in response to stimulation of all stimulus combinations for one AMI site at a time. The recording ground wire was positioned under the skin roughly $2 \mathrm{~cm}$ rostral to bregma.

\section{Placement of probes}

Post-stimulus time histograms (PSTHs) and frequency response maps (FRMs) were plotted online to confirm that the arrays were correctly positioned along the tonotopic axis of the ICC (Fig. 2A) and along the appropriate BF columns in A1 (Fig. 2B). Details on PSTH, FRM, and BF calculation are presented in a previous publication (Lim and Anderson 2006). Briefly, we bandpass-filtered the neural signals (300$3,000 \mathrm{~Hz})$ and detected spikes on each site that exceeded 3.5 times the standard deviation of the background noise signal. We binned the spikes into PSTHs ( $1 \mathrm{~ms}$ bins). The number of trials for broad-
A

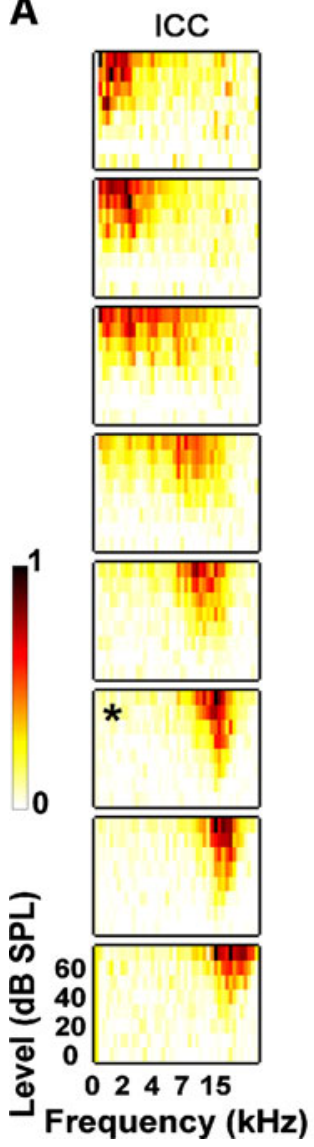

B

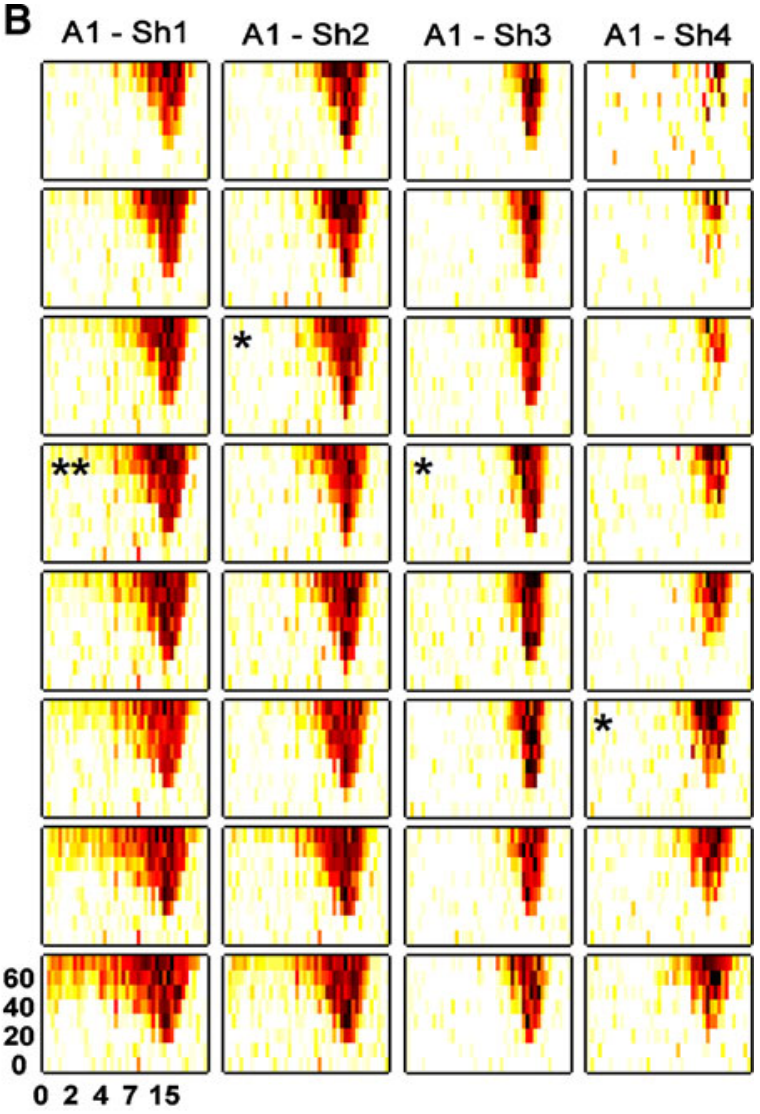

FIG. 2. Frequency response maps (FRMs) recorded on 8 different sites (only 8 of 20 sites shown) along the tonotopic gradient of the central nucleus of the inferior colliculus (ICC) with the AMI array (A) and on 32 different sites within a specific frequency region $(\sim 16 \mathrm{kHz})$ of the primary auditory cortex $(A 1)$ with a multi-site probe (4 shanks each with 8 sites) (B). For each FRM, the $x$-axis is frequency $(0-30 \mathrm{kHz}, 8$ steps/octave) and the $y$-axis is stimulus intensity (0-70 dB SPL, $10 \mathrm{~dB}$ steps). The color scale corresponds to normalized driven spike rate where all negative FRM values were set to zero to improve visualization. The FRM labeled with an asterisk in A corresponds to the selected AMI site (16 kHz region) used for electrical stimulation in the ICC. The FRMs in B with an asterisk correspond to sites located within the main input layer of A1 and the double asterisk represents the site selected for analysis since it had a best frequency closest to that of the ICC site. 
band stimulation varied, but for the FRMs, we presented each pure tone four times. We then calculated the driven spike rate (total minus spontaneous activity) within a set PSTH window relative to the stimulus onset (A1 5-25 ms, ICC 5-65 ms) and plotted the value for each stimulus to create the FRM for each site as displayed in Figure 2. The BF was taken as the centroid value at $10 \mathrm{~dB}$ above the level where we first observed a noticeable and consistent response.

For A1 probe placement, we first recorded surface potentials in response to pure tones using the tip site of each shank and determined the borders of A1 based on its BF organization (Wallace et al. 2000). This minimized insertion damage within A1. We then inserted and aligned the A1 probe in a specific BF region in which each shank was approximately aligned along a cortical column. The A1 sites usually exhibited onset responses. Obtaining FRMs with approximately similar BFs across sites, as shown in Figure 2B, confirmed that our probe was correctly positioned in A1. To identify the recording site along each shank that was located in the main input layer of A1, we performed current source density analysis (Muller-Preuss and Mitzdorf 1984; Mitzdorf 1985; Kral et al. 2000) in response to $70 \mathrm{~dB}$ SPL broadband noise (100 trials) using the finite difference formula:

$$
\operatorname{CSD}(z)=\sigma_{z} \frac{\phi(z+\Delta z)-2 \phi(z)+\phi(z-\Delta z)}{(\Delta z)^{2}}
$$

where $\phi$ is the averaged evoked potential, $z$ is the depth location of each site along an A1 probe shank, $\Delta z$ is the differentiation step size, and $\sigma_{z}$ is the component of conductivity in the $z$-direction. $\Delta z$ was equal to our A1 site spacing of $100 \mu \mathrm{m}$ and $\sigma_{z}$ was set to one since we were not concerned with absolute CSD values. CSD profiles for the two edge sites could not be calculated using the above equation since it requires evoked potential profiles from two neighboring sites. The one-dimensional current source density approximation provides a consistent representation for the current sinks and sources associated with columnar synaptic activity in the auditory cortex. The main input layer of $\mathrm{A} 1$, which approximately corresponds to layers III/IV (Huang and Winer 2000; Smith and Populin 2001), was taken as the site with the shortest latency current sink and PSTH response (asterisks in Fig. 2B). Details on and results using this method have been presented previously (Lim and Anderson 2007b; Middlebrooks 2008). Of the four identified A1 sites, we only selected one site with the closest BF to the ICC site BF for further analysis (double asterisk in Fig. 2B). In most cases, the BFs were not identical across A1 sites. However, in cases where multiple sites had the same BF, we used the recording site with the shortest latency and largest magnitude response to acoustic stimulation.

For AMI array placement, we initially inserted a Michigan probe with two shanks (eight sites along each shank) to locate the ICC. This initial procedure avoided excessive insertions of the AMI array, which has a greater diameter than the Michigan probe ( $0.4 \mathrm{~mm}$ versus $0.015 \mathrm{~mm})$, that could induce additional damage to the IC. After identifying an appropriate trajectory, the AMI array was inserted into the ICC. We typically observed sustained PSTHs in response to broadband noise and, as shown in Figure 2A, FRMs that exhibited an orderly shift in BF (Snyder et al. 2004; Lim and Anderson 2006).

Figure 2 presents typical examples of FRMs recorded in the ICC and A1. We discontinued any experiments in which we observed obvious elevations in thresholds across the FRMs indicating abnormal hearing in our animal.

\section{ICC probe histology and site locations}

Before the first placement of the AMI array into the brain, it was dipped into a red stain $(3 \mathrm{mg}$ Di-I per $100 \mu \mathrm{L}$ acetone; Di-I: 1,1-dioctadecyl-3,3,3', $3^{\prime}$-tetramethylindocarbocyanine perchlorate; Molecular Probes, Eugene, OR, USA) to later identify the different placements (DiCarlo et al. 1996; Lim and Anderson 2007a). At the end of the experiment, the animal was decapitated and the head was immersed in $4 \%$ paraformaldehyde for 1 day. Afterwards the brain was removed and fixed again in paraformaldehyde for approximately 14 days. The portion of the brain consisting of the right superior and inferior colliculi was dissected. To have a fixed point for later comparisons across animals, a reference point (RP) was created by inserting a Di-I-dipped needle in the rostral intersection of the IC and superior colliculus (SC) border (Fig. 3B, plus symbol). The dissected brain was then placed into $20 \%$ sucrose solution until the tissue sank (24 h). Frozen sagittal sections (sliced at $-20^{\circ} \mathrm{C}$ ) with a thickness of $40 \mu \mathrm{m}$ were prepared and analyzed using a stereomicroscope (magnification of $\times 10$; Nikon SMZ1500, Nikon Instruments, Melville, NY, USA) and a digital camera system (Colorview XS, Software Analysis ${ }^{\circledR}$, Soft imaging Systems GmbH, Münster, Germany) (Fig. 3C).

In each section, the track of the AMI array appeared as a dot since we inserted the array at an angle of $45^{\circ}$ from the sagittal plane. To identify the location of the AMI tracks within the ICC, we needed to develop a coordinate system that would be approximately consistent across animals. The orientation of the brain shown in Figure $3 \mathrm{~A}$ and $\mathrm{B}$ corresponds to how the brain, when first removed from the skull without yet dissecting out the IC-SC portion, lies on a 

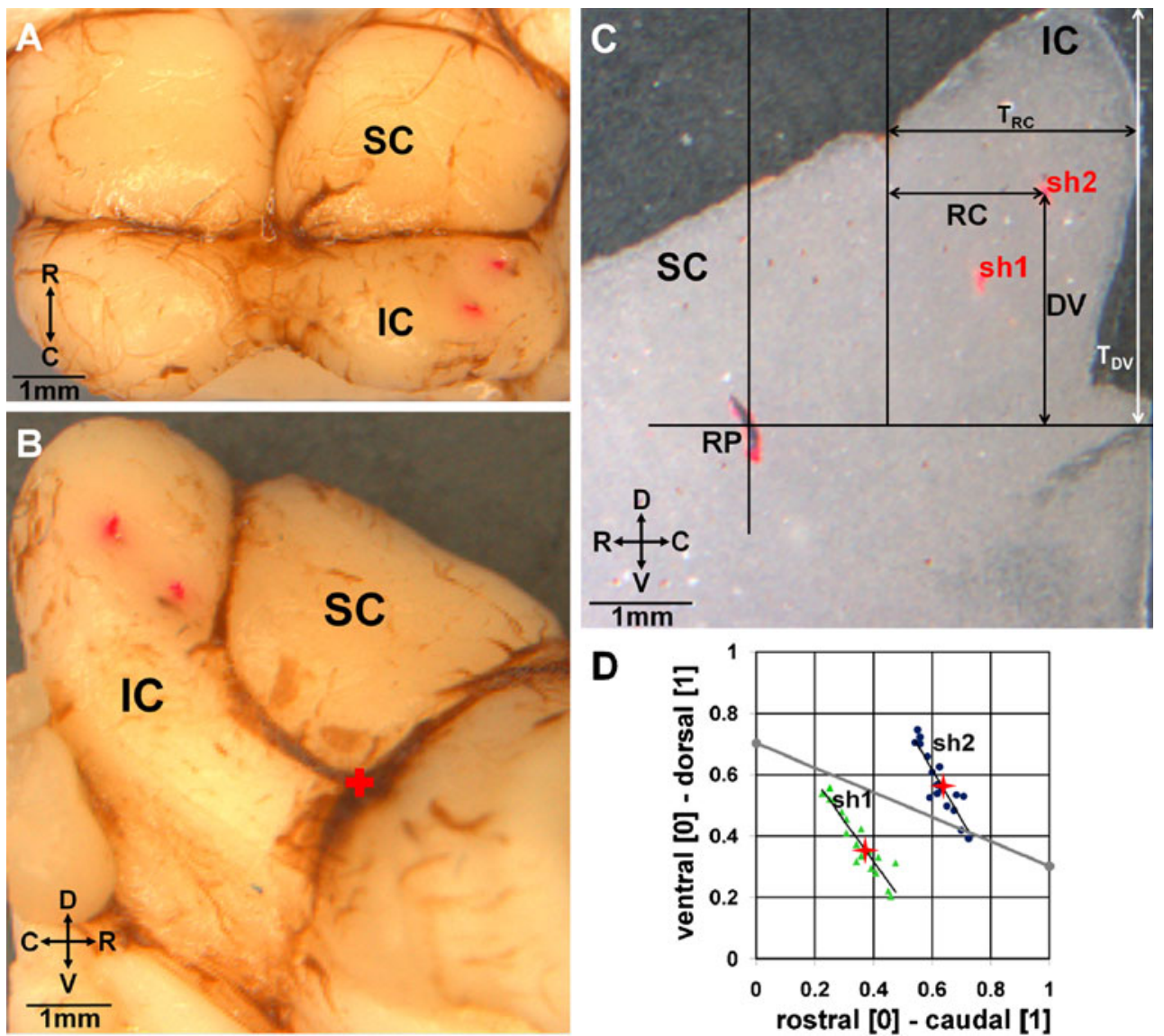

D

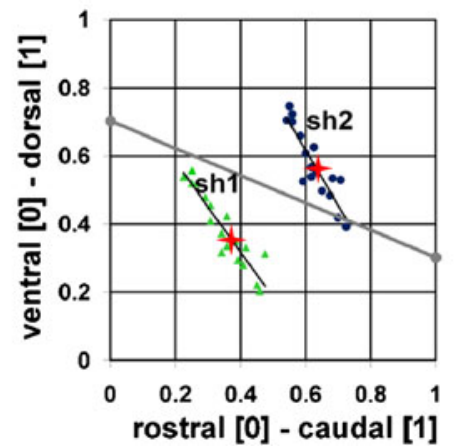

FIG. 3. Reconstruction of the AMI array placements in the inferior colliculus $(I C)$ for one animal. A View of the dorsal surface of the IC and superior colliculus (SC). The two red dots indicate where we inserted the AMI array. B Lateral view of the right IC and SC in which the red plus sign represents the location of our reference point $(R P)$ used for normalizing the array locations across animals. C A 40- $\mu \mathrm{m}-$ thick sagittal slice detailing our coordinate system used to quantify the location of the trajectory for each array placement (appears as dots in each slice since the array was inserted at a $45^{\circ}$ angle to the sagittal plane). D The normalized coordinates (RC divided by $T_{\mathrm{RC}}$, DV divided by $T_{\mathrm{DV}}$ ) are plotted across several slices (dots) for each

flat surface. The vertical line going through RP in Figure $3 \mathrm{C}$ is parallel to the cutting plane of the brain used to dissect the IC-SC portion from the rostral half of the brain. The horizontal line through RP is drawn perpendicular to that vertical line. We then are able to draw parallel vertical lines from the rostral corner of the IC (IC-SC border) as well as at the caudal end of the IC (white line) as shown in Figure 3C.

Along the caudal-rostral dimension we normalized $\mathrm{RC}$, which is the distance between the track point and the IC-SC border, by $T_{\mathrm{RC}}$. $T_{\mathrm{RC}}$ is the total distance between the caudal edge of the IC and the IC-SC border. Along the dorsal-ventral dimension we normalized DV, which is the distance from the track point to the horizontal line through $\mathrm{RP}$, by $T_{\mathrm{DV}} . T_{\mathrm{DV}}$ is the distance between the dorsal tip of the IC and the horizontal line through RP. Since these parameters

trajectory (placement 1: green, placement 2: blue). Since these coordinates systematically change across slices, we only selected the values for the slice corresponding to 0.54 from the lateral edge to the midline (red pluses) that were then later displayed on a single plot across all animals. The red pluses in $\mathbf{D}$ correspond to the red dots caused by the track of the AMI in $\mathbf{C}$. The gray line in $\mathbf{D}$ divides which locations we labeled as caudal (above the line) and rostral (below the line) and was based on the threshold values shown in Figure 6. For further details on our reconstruction and grouping methods, see Methods: "ICC probe histology and site locations". C caudal, D dorsal, $R$ rostral, $V$ ventral.

systematically varied across sagittal slices (Fig. 3D), we selected only the normalized values for the slice corresponding to a distance of approximately 0.54 from the lateral edge of the IC to the midline of the brain for comparative analysis across animals (red pluses in Fig. 3D).

There are limitations in this measurement procedure in that it does not provide an exact location along a true isofrequency lamina, which varies in curvature and dimensions depending on the $\mathrm{BF}$ region (Malmierca et al. 1995). Our approach assumes that the laminae are flat layers aligned $45^{\circ}$ below the horizontal plane. There are also slight variations in brain dimensions across animals. However, the benefit of this method is that it is possible to visualize and assess different response properties as a function of location within the IC using a consistent 
coordinate system. The normalization procedure also minimizes the effects of varying tissue shrinkage across preparations and differences in overall brain dimensions. As shown in the results below, we pooled all the data across BF regions and animals into one generalized isofrequency lamina in which all the track points (corresponding to the pluses as shown in Fig. 3D) were plotted onto one figure. At least with this mapping and pooling method, it is possible to claim that if we observe any systematic shifts in neural properties with AMI stimulation location, then it must generally exist along the isofrequency domain of the ICC for the BF regions we tested. Otherwise, we would not observe the trends when pooling and essentially averaging out the effects across $\mathrm{BF}$ regions and animals. If we do not observe any systematic shifts, we cannot rule out the possibility that trends were masked by the errors introduced by our methods.

We did observe location trends for some coding properties that varied from the caudal-dorsal region to the rostral-ventral region of the ICC. To further compare properties between these two regions, we pooled the data into a "caudal" and a "rostral" group depending on if the locations were caudal (above) or rostral (below) to the gray diagonal line in Figure 3D. This line was determined based on visual assessment of Figure 6A, which also includes a diagonal gray line that separates the locations into those with higher and those with lower thresholds. Note that the coordinates of the contour plots have been reversed from those in the histological reconstruction in Figure 3D to enable direct comparison to previously published plots (Lim and Anderson 2007a) and to be consistent with how these laminae would be observed if correctly viewing the IC from the right side of the animal. The purpose of this grouping is not to claim that there are exactly two distinct regions based on our division, but just to provide a simplified way to visualize how different properties later analyzed in this paper vary for different "caudal" versus "rostral" ICC regions.

\section{Data analysis}

We analyzed the evoked potentials recorded on an A1 site in response to stimulation of an AMI site, in which both sites had a similar BF. We averaged the evoked potentials across 20 trials for each PD and level. Figure 4 presents typical evoked potential curves for three different PDs and two levels of stimulation for one AMI site. The stimulus artifact occurred at $0 \mathrm{~ms}$. The evoked potentials generally had a similar shape across stimuli and their amplitudes increased with both PD and level. For analysis, we smoothed the averaged curves (filtering of $30-7500 \mathrm{~Hz}$ ) to more consistently calculate the magnitude (relative to $0 \mathrm{mV}$ ) and latencies (relative to stimulus onset at $0 \mathrm{~ms}$ ) of the negative peak. Electrical threshold for A1 activation was defined as the highest level where the evoked potential could not be visually detected from the spontaneous activity.

Steepest gradient axis. The values for threshold, peak magnitude, and peak latency and across AMI stimulation locations and animals were plotted as

\section{PD: $123 \mu s$}
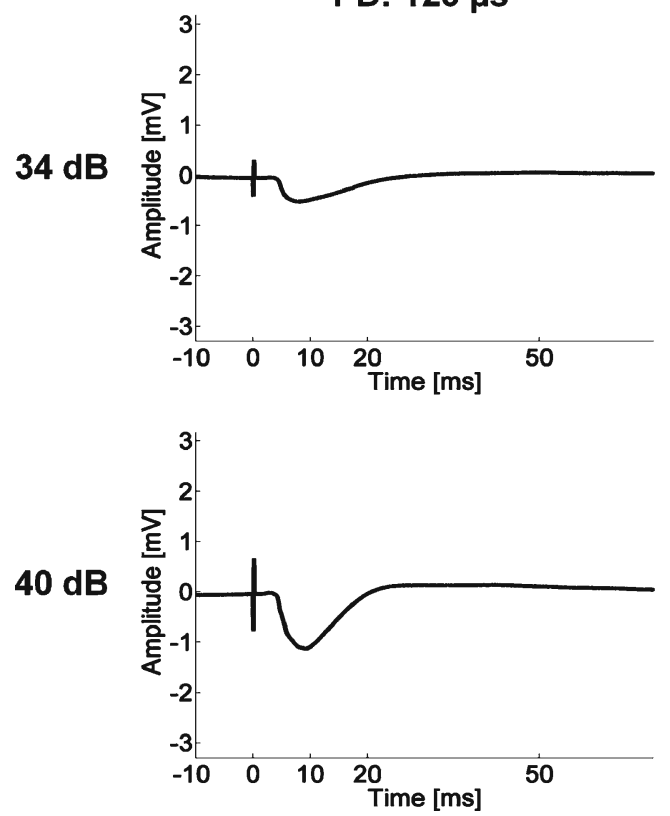

PD: $328 \mu s$
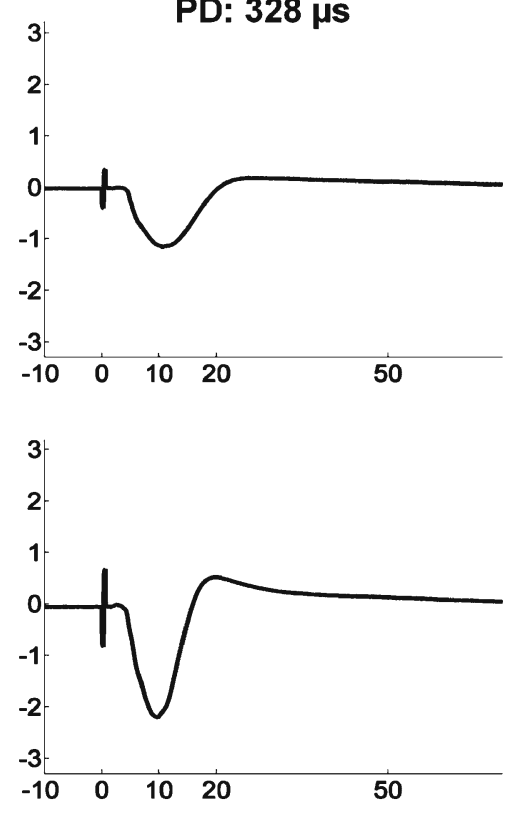

PD: $984 \mu s$
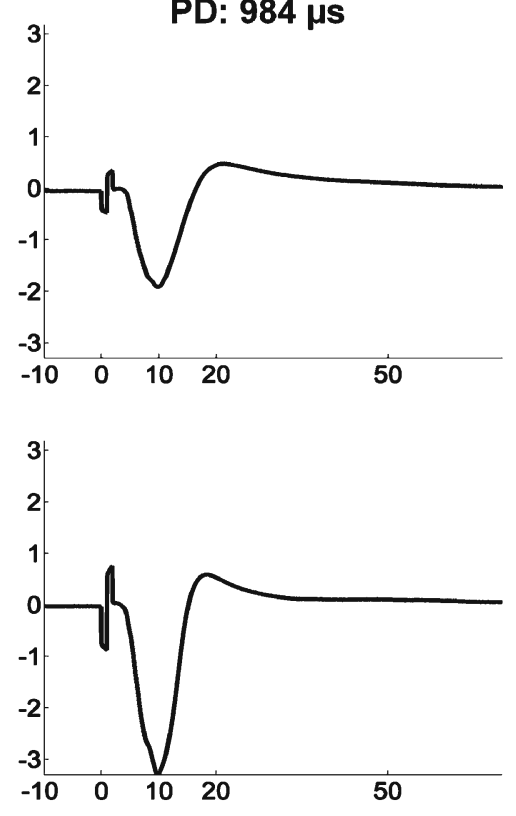

FIG. 4. Averaged evoked potentials (20 trials) recorded on the main input layer site in primary auditory cortex in response to stimulation with an $\mathrm{AMI}$ site for three different phase durations $(P D s)$ and two current levels. The sites are located within a 16-kHz region. The stimulus started at 0 ms and elicited an electrical artifact that systematically changed with PD and level. 
contour plots representing a generalized isofrequency lamina (see Figs. 6 and 7 for examples). To assess if there were any systematic trends in these parameters with stimulation location, we first determined the steepest gradient axis through all the points. This was achieved by selecting a reference point (the circle on each contour plot is an example of one reference point) and drawing a line through the data points within the contour plot with a certain angle. The data points were then collapsed onto a two-dimensional plot relative to this line in which the parameter value was plotted against the location relative to the reference point (for example, bottom of Fig. 6A). A line was drawn through the collapsed data using a linear least-squares fit and the slope of that line was determined. We repeated this procedure for all reference points and angles to obtain the case in which the largest slope value occurred, providing the steepest gradient axis. We could then plot a given parameter along this steepest gradient axis to determine if it significantly changed as a function of ICC stimulation location.

\section{RESULTS}

For this study, data were obtained from nine animals. In each guinea pig, we electrically stimulated one to four locations in the ICC and recorded the corresponding neural activity in A1. In total, we had 21 stimulation locations across all animals in which the BF could vary for different sites (BFs 1.2, 1.5, 1.5, 1.7, 1.8, 2.0, 2.3, 3.5, 6, 10, 10, 11, 11, 16, 16, 16, 17, 19, 27, 27 , and 30 ). The BFs listed were arbitrarily rounded to the nearest hertz above $4 \mathrm{kHz}$ and nearest tenths of a hertz below $4 \mathrm{kHz}$. We usually did not have identical BFs for both the site in the ICC and A1 though they were quite similar. The actual discrepancy ranged from 0.002 to 0.348 octaves (mean 0.118, SD 0.110). In total, we had 12 caudal and 9 rostral ICC stimulation locations (for details on how we obtained this grouping, see Methods: "ICC probe histology and site locations"). We use the terms "caudal" and "rostral" throughout the text to refer to these groupings. In cases where we refer to a specific location along the isofrequency domain of the ICC, it will be clarified in the text. Table 1 presents how many of these caudal and rostral locations were presented with a given PD. Only PDs of 41 and $123 \mu$ s were used for all locations. The variability in PDs used for each location was due to our initial efforts to select a sufficient range of PDs for our study, in which only a few could be used for a given experiment due to time constraints.

Additionally, we present data from a different series of experiments in which two single shank arrays were simultaneously positioned into a caudal and a rostral ICC region providing two sites within a similar isofrequency lamina but $1.5 \mathrm{~mm}$ apart. A total of 15 of these site pairs across 8 animals were available. The BFs ranged between 1.4 and $18 \mathrm{kHz}$ with an ICC-to-A1 BF discrepancy between 0 and 0.415 octaves (mean 0.089, SD 0.096).

\section{Threshold versus phase duration}

In Figure 5, the thresholds versus the different PDs are plotted for all 21 ICC locations. In general, we observed a decrease in threshold with longer PDs when plotted in microamperes (Fig. 5A). When

TABLE 1

Summary of phase durations (PDs), number of locations for each PD and group in the central nucleus of the inferior colliculus (ICC), and their mean threshold values including standard deviations

\begin{tabular}{|c|c|c|c|c|}
\hline \multirow[b]{2}{*}{$P D[\mu s]$} & \multicolumn{2}{|c|}{ Number of locations } & \multicolumn{2}{|c|}{ Mean threshold (SD) $[\mu \mathrm{A}]$} \\
\hline & Rostral & Caudal & Rostral & Caudal \\
\hline 41 & 9 & 12 & $100.2(38.4)$ & $236.0(168.7)^{*}$ \\
\hline 82 & 6 & 8 & $48.5(14.6)$ & $166.3(146.1)^{*}$ \\
\hline 123 & 9 & 12 & $35.6(13.5)$ & $102.6(89.4)^{*}$ \\
\hline 205 & 6 & 8 & $26.6(9.6)$ & $90.2(85.6)^{*}$ \\
\hline 328 & 9 & 8 & $20.9(9.5)$ & $48.5(46.5)$ \\
\hline 410 & 0 & 4 & & $61.4(65.5)$ \\
\hline 492 & 3 & 1 & $11.0(2.3)$ & 20.0 \\
\hline 656 & 6 & 7 & $18.1(5.8)$ & $31.9(22.9)$ \\
\hline 820 & 0 & 4 & & $45.9(53.6)$ \\
\hline 984 & 6 & 7 & $15.1(5.1)$ & $25.4(18.2)$ \\
\hline 1476 & 3 & 4 & $12.5(7.5)$ & $16.3(7.5)$ \\
\hline
\end{tabular}

For more details on our grouping method, see Methods: "ICC probe histology and site locations"

$* P<0.05$ indicates significant differences between rostral versus caudal values based on a Welch's $t$ test 

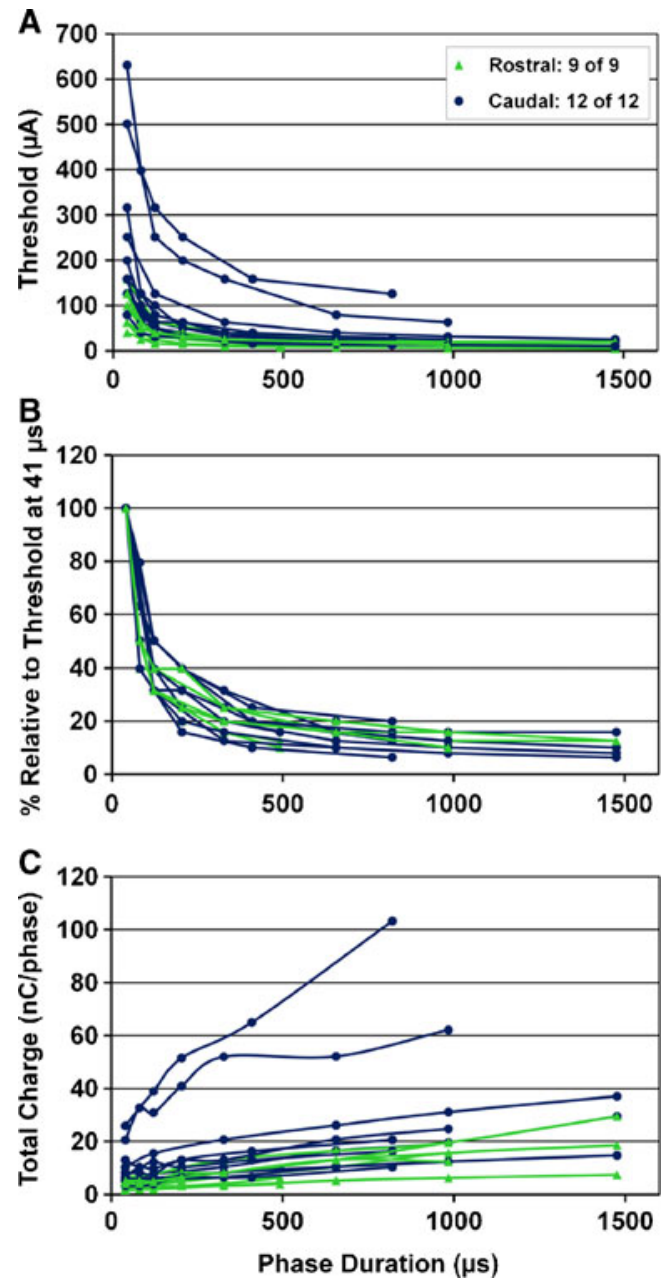

FIG. 5. Threshold versus phase duration curves for different stimulation locations within the central nucleus of the inferior colliculus. Thresholds are plotted in microamperes (A), in percentage relative to the threshold at $41 \mu \mathrm{s}(\mathbf{B})$, and total charge per phase $(\mathbf{C})$. The locations are divided into rostral (green) and caudal (blue) groups (for more details on the grouping method, see Methods: "ICC probe histology and site locations"). Different phase durations were used across curves (see Table 1).

plotting threshold as total charge per phase, thus total energy per phase, the thresholds actually increased with PDs (Fig. 5C). This was consistent for both caudal (blue circles) and rostral (green triangles) locations. However, stimulation of caudal locations exhibited a wider range of threshold values that were usually higher than those for stimulation of the rostral region. In Figure 5C, it can be seen that for two caudal locations, activation thresholds required substantially greater energy than those for the other locations. This is interesting in that all the curves correspond to locations within the ICC yet they can exhibit dramatically different activation effects to electrical stimulation with the AMI array. By normalizing the curves to the threshold value at $41 \mu$ s (Fig. $5 \mathrm{~B}$ ), it is possible to observe that the curves generally exhibit a similar shape with the largest drop in threshold current between 41 to $123 \mu$ s that flattens out at roughly $500 \mu \mathrm{s}$. Overall, these results indicate that shorter PDs can reduce energy requirements (i.e., total charge) for neural activation but at the cost of higher required current levels, which is usually limited by the power supply and safety features of an implant device. A balance between total energy and current level appears to be achieved by using a PD around $123 \mu \mathrm{s}$, which is the point where current level has dropped dramatically.

\section{Threshold versus location}

The strength-duration curves presented in Figure 5 revealed large differences in thresholds between rostral and caudal locations. To better depict these differences, we plotted contour plots consisting of the threshold values for each stimulation site as a function of location along the two-dimensional isofrequency domain of the ICC for several PDs (Fig. 6). The coordinates of these contour plots have been reversed from those in the histological reconstruction (Fig. 3D) to enable direct comparison to previously published plots (Lim and Anderson 2007a) and to be consistent with how these laminae would be observed if correctly viewing the IC from the right side of the animal. Across all PDs, we observed a general trend of higher to lower thresholds when stimulating from caudaldorsal to rostral-ventral locations, respectively, in the contour plots. For clarity, we identified the steepest gradient axis (see Methods: "Data analysis") and collapsed the contour plots into two-dimensional plots of threshold versus location along the steepest gradient axis (black line in each contour plot in which location is relative to the open circle). Thresholds were consistently and significantly lower in more rostral-ventral regions. The plots for a PD of 41 and $123 \mu$ s consist of all 21 locations and exhibit clear negative trend lines $(P<0.001)$ that account for $61 \%$ of the variation in the data. For longer PDs, the trend was less clear partly due to fewer locations available for each plot. However, the plots for all PDs in Figure 6 still exhibit significantly negative trend lines $(P<0.05)$.

As shown in Figure 6 (in decibel) and Table 1 (in microamperes), there were large differences in threshold values between rostral and caudal regions. For example, for a PD of $41 \mu$ s (Fig. 6A), the largest threshold value, which was in the caudal region, was about $56 \mathrm{~dB}(631 \mu \mathrm{A})$ while the smallest value, which was in the rostral region, was about $32 \mathrm{~dB}(40 \mu \mathrm{A})$ resulting in a difference of $24 \mathrm{~dB}$. In terms of mean threshold values (Table 1), the rostral region consisted of values more than two times greater than 
A
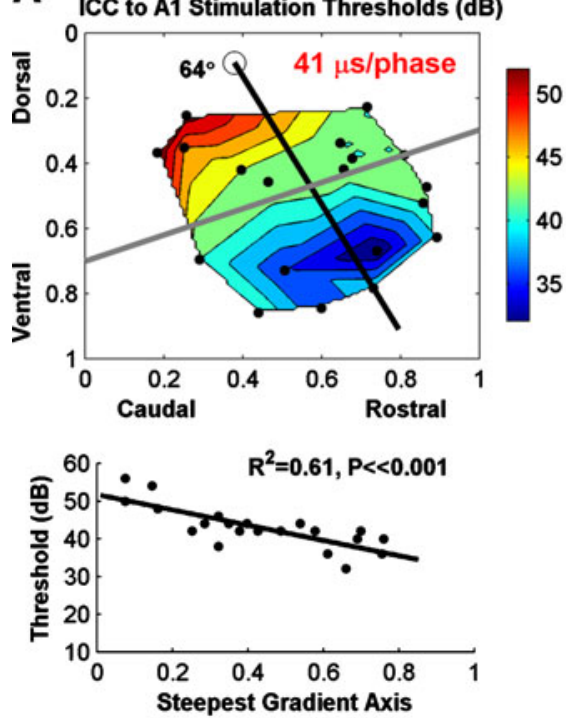

D
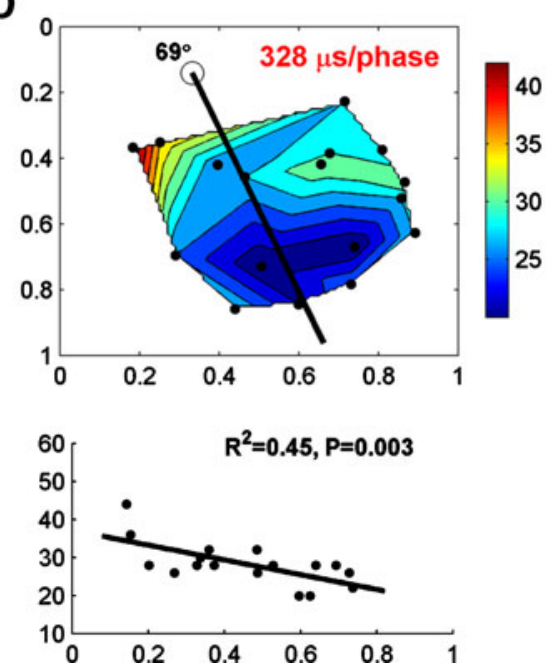

B
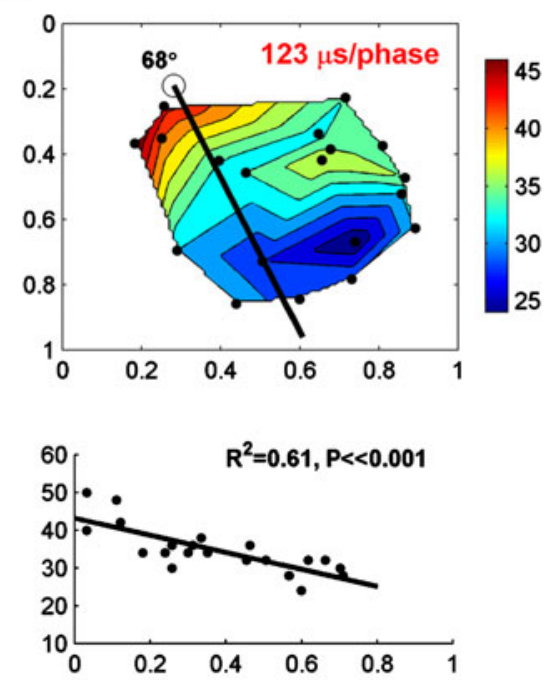

E
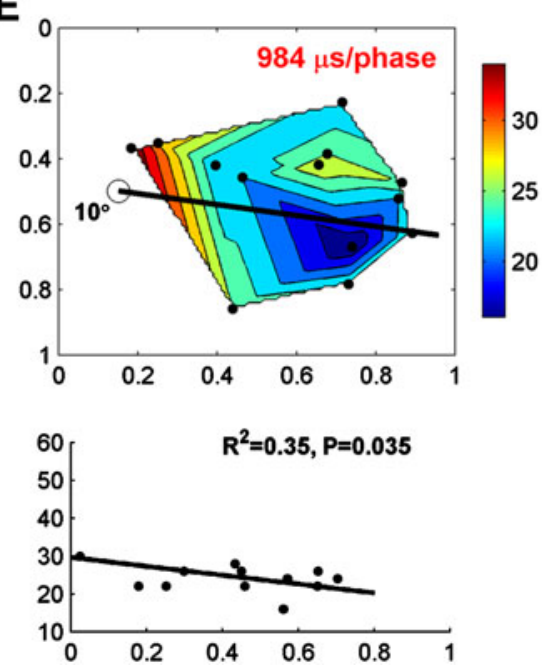

C
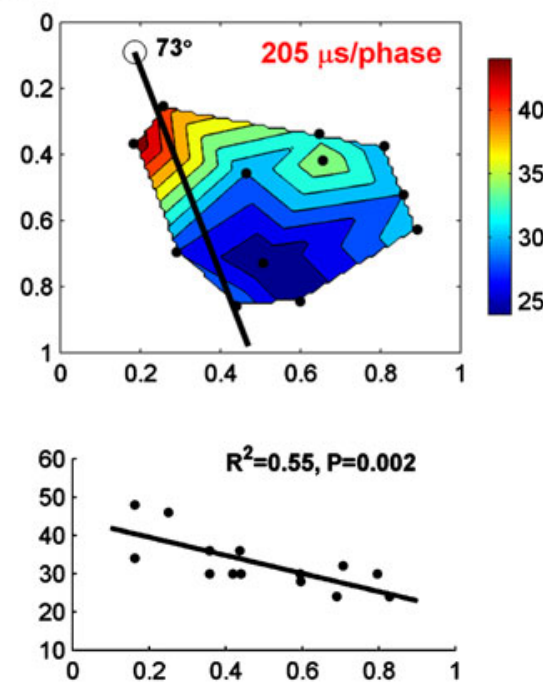

FIG. 6. Contour plots of activation thresholds in the primary auditory cortex (A1) as a function of stimulation location along the normalized isofrequency domain of the central nucleus of the inferior colliculus (ICC). Each pair of plots (A-E) corresponds to a different phase duration $(P D)$. Each dot corresponds to a different stimulation site. In each contour plot, a black line is present that corresponds to the steepest gradient axis for threshold values, as depicted in the plot below that shows threshold versus location along that steepest axis (at an angle below the horizontal line; 0 along the steepest gradient axis corresponds to the location of the circle in the contour plot). For all PDs, there was a significant trend of lower thresholds for more rostral-ventral locations of the ICC. For longer PDs, this trend became less clear though maintained significance $(P<$ 0.05). The gray line in $\mathbf{A}$ was based on our visual determination of

those for the caudal region for PDs less than or equal to $328 \mu \mathrm{s}$. This threshold difference became smaller for longer PDs. This threshold difference also became less significant $(P>0.05)$ for longer PDs, which is partly due to the limited number of points. In addition to higher thresholds, the caudal region also exhibited greater variation in thresholds (i.e., standard deviation values) compared to the rostral region the midpoint of the threshold gradient along this caudal-dorsal to rostral-ventral gradient in which a reasonable number of caudal and rostral locations were separated into two groups for our analyses. The color scale of the contour plots corresponds to threshold levels in decibel relative to $1 \mu \mathrm{A}$. Only PDs of 41 and $123 \mu$ s consist of all 21 locations while the other PDs consist of a subset of these points (see Table 1). Not all points were visible since two locations consisted of two points with identical coordinates $([0.26,0.25]$ and $[0.65,0.34]$; stimulation of two different frequency regions for the same AMI array placement). For example, in A only 19 of the 21 points are visible. The threshold values for each pair were averaged for the contour plots. For more details on how we identified the different ICC locations or calculated the steepest gradient axis, see "Methods".

that further suggests that there are differences in functional activation properties between these two regions.

We also verified our results across a series of animals in which we simultaneously inserted two shanks into a caudal and rostral region and then stimulated one site from each shank located within the same BF lamina. At a PD of $205 \mu$ s, stimulation of 
the rostral site achieved a lower threshold for A1 activation in 14 of the 15 cases (a threshold difference ranging from 3 to $59 \mu \mathrm{A}$ ). In one case, the thresholds were identical. Across the 15 rostral ICC locations, we obtained a mean threshold of $16.75 \mu \mathrm{A}$ (SD 4.8), and for the 15 caudal locations, a mean threshold of $36.56 \mu \mathrm{A}$ (SD 17.7). The threshold difference was significant based on a Student's paired $t$ test $(P<<$ 0.001). The overall thresholds across both regions were lower in these animals than our presented results above. This was expected since we intention- ally, for other experimental purposes, inserted the arrays more rostrally to avoid the most caudal-dorsal edge where we observed the highest thresholds.

\section{Magnitude versus location}

Similar to thresholds, we assessed how the evoked potential magnitudes recorded in the main input layer of A1 were affected by stimulation location and phase duration in addition to current level. In Figure 7, the evoked potential magnitudes are plotted
A ICC to A1 Stimulation Magnitudes (mV)

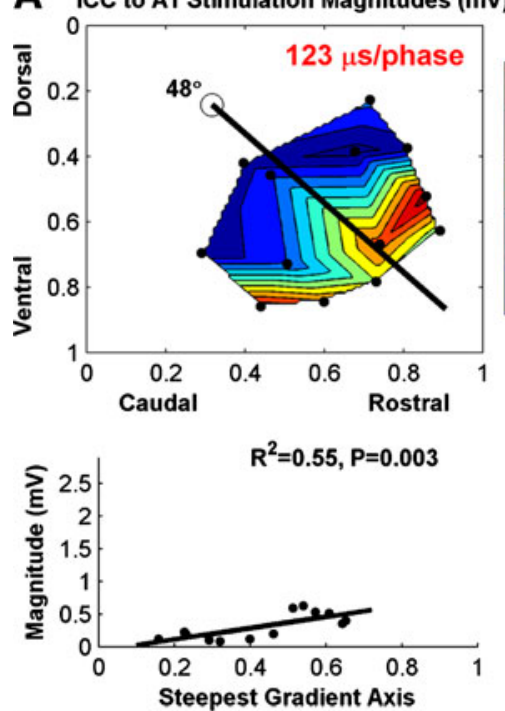

D

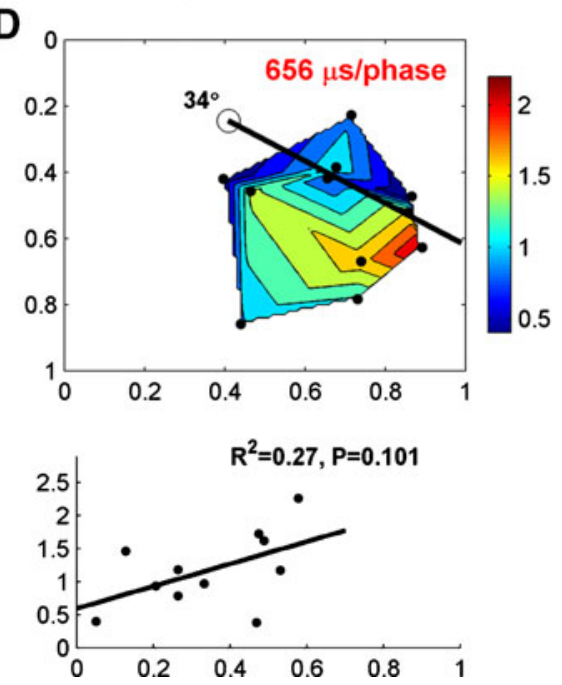

FIG. 7. Contour plots of evoked potential magnitudes in the primary auditory cortex $(A 1)$ as a function of stimulation location along the normalized isofrequency domain of the central nucleus of the inferior colliculus (ICC) for one stimulus level of $40 \mathrm{~dB}$ relative to $1 \mu \mathrm{A}$. Each pair of plots (A-E) corresponds to a different phase duration $(P D)$. Each dot corresponds to a different stimulation site. In each contour plot, a black line is present that corresponds to the steepest gradient axis for peak magnitude values, as depicted in the plot below that shows magnitude versus location along that steepest axis (at an angle below the horizontal line; 0 along the steepest gradient axis corresponds to the location of the circle in the contour plot). For all PDs, there was a
C
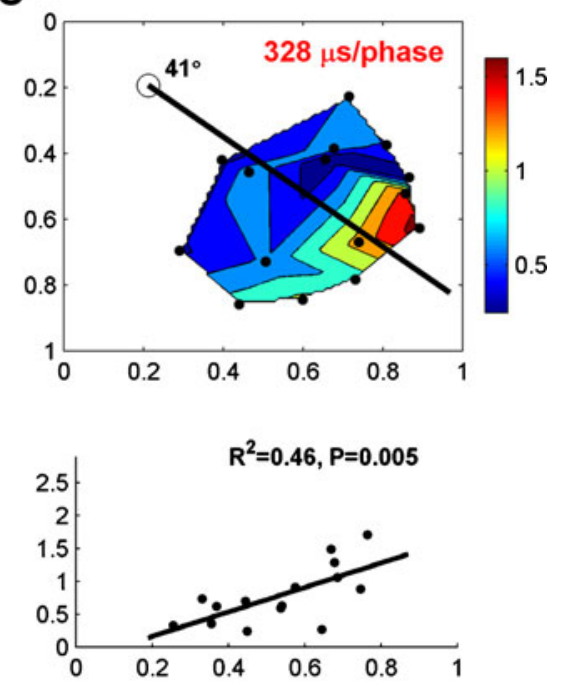

E

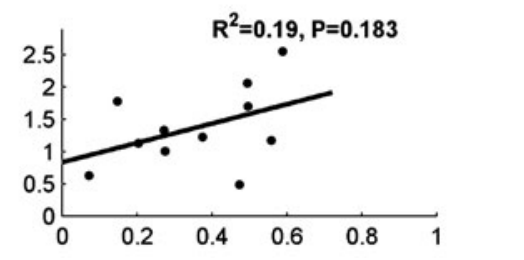

significant trend of higher magnitudes for more rostral-ventral locations of the ICC. For longer PDs (656 and $984 \mu \mathrm{s})$, this trend was no longer significant $(P>0.05)$. The color scale of the contour plots corresponds to magnitude values in millivolts $(\mathrm{mV})$. Not all the same locations are used for each plot since different phase durations were used for different sites and animals (see Table 1). The most caudaldorsal locations are not included because no visible activity was present at $40 \mathrm{~dB}$. This specific level was selected to present one example of how magnitude values change with ICC stimulation location. For more details on how we identified the different ICC locations or calculated the steepest gradient axis, see "Methods". 
versus ICC location in response to a stimulation level of $40 \mathrm{~dB}$ (equal to $100 \mu \mathrm{A}$ ). We only present one level to show how the magnitudes change with stimulation location. The overall effects of level on the magnitude values are presented in Figure 8 . At $40 \mathrm{~dB}$, the shorter PDs did not elicit sufficient activity across many of the more caudal-dorsal locations; thus, we present contour plots for higher PDs: 123, 205, 328, 656, and
A

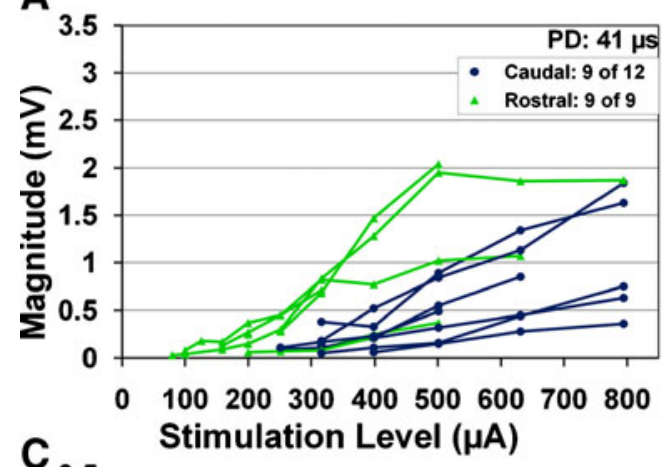

$\mathrm{C}_{3}$

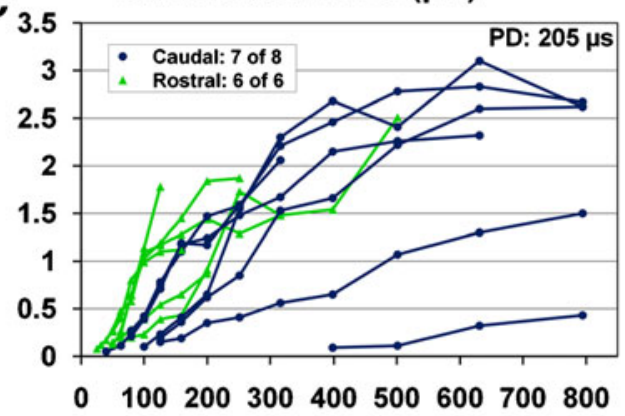

E

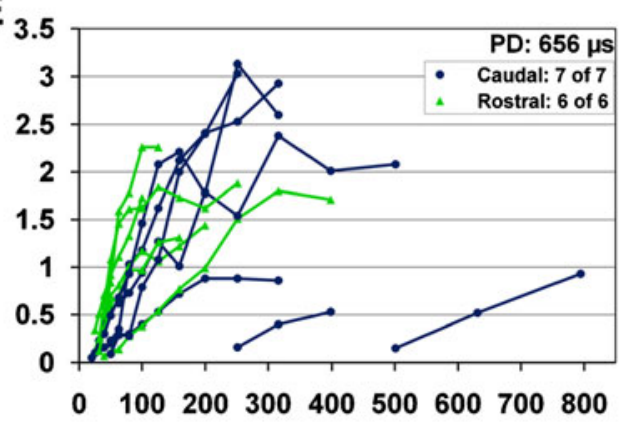

$\mathrm{G}_{3.5}$

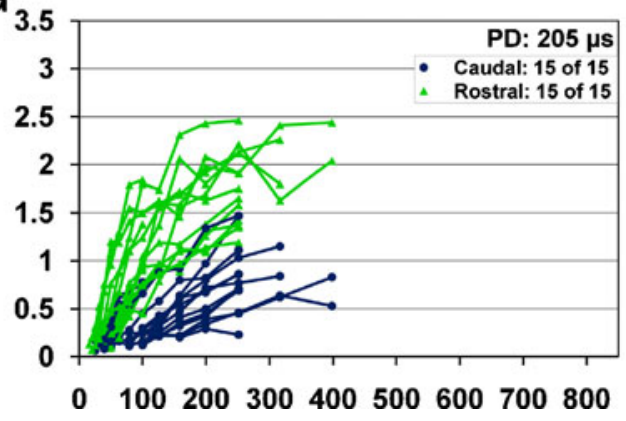

FIG. 8. Evoked potential magnitudes recorded in the primary auditory cortex as a function current level for different stimulation locations in the central nucleus of the inferior colliculus (ICC) and phase durations $(P D s)(\mathbf{A}-\mathbf{E})$. Some of the caudal locations listed in Table 1 were not included in some of these plots since the elicited evoked potentials were not large enough to accurately measure the peak magnitude though the threshold of activation could still be
B
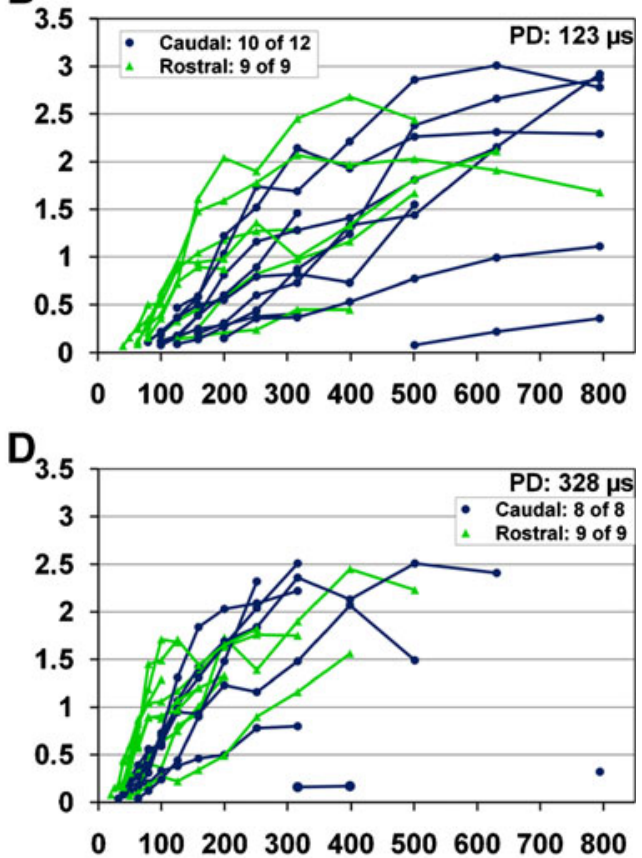

$F_{3.5}$

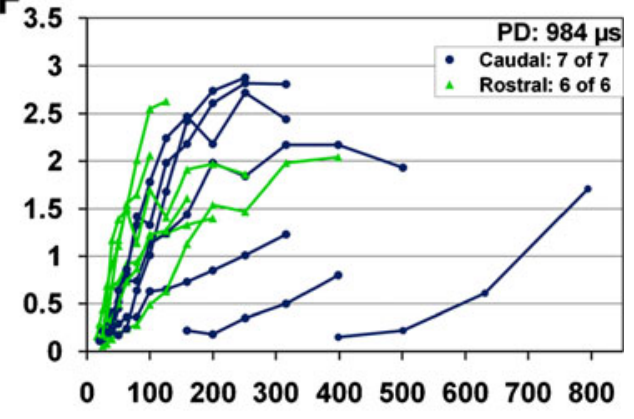

determined (see legend on each plot). G Data for another series of experiments in which two shanks were simultaneously inserted into the ICC but one within a caudal region and another within the rostral region to ensure two sites within the same best frequency lamina but in different isofrequency locations. Color corresponds to the two different groups we used for analysis. For details on how we grouped the sites, see Methods: "ICC probe histology and site locations". 
$984 \mu \mathrm{s}$. For the most caudal-dorsal locations, no activity was elicited across PDs, and therefore, those locations were not included in the plots.

In general, the magnitude values in each plot increased with longer PDs. Consistent with Figure 6, we observed a shift in values from the caudal-dorsal region to the rostral-ventral region in which magnitudes became larger. This trend is clearly depicted for PDs of 123, 205, and $328 \mu$ s. However, at the longer PDs of 656 and $984 \mu \mathrm{s}$, this trend is no longer significant. Although the lack of significance can be partly attributed to fewer points distributed across the contour plot, it also appears that stimulation with longer PDs creates a more complex pattern of magnitude values across the isofrequency domain of the ICC. The most caudal-dorsal (especially those sites that elicited no activity thus were not included in the plots) and rostral-ventral regions still have the smallest and largest evoked potential magnitudes, respectively. However, it appears that stimulation of more central locations (along the caudal-dorsal to rostral-ventral axis) exhibits a wider range of values for longer PDs.

\section{Magnitude growth functions}

From Figures 6 and 7, one question that arises is if the magnitude contour plots are a direct result of the threshold contour plots. In other words, are smaller evoked potentials elicited by stimulating more caudal-dorsal regions because activation thresholds are much higher in those regions? This is an important question for AMI implementation because if the magnitudes are smaller for a constant stimulus level simply because the thresholds are higher, then it may be possible to effectively stimulate caudal-dorsal locations by increasing current levels. However, if the magnitudes are small because their growth functions with stimulus level are extremely shallow or saturate at smaller evoked potential magnitudes, then it may not be possible to effectively activate caudal regions within the range of current levels dictated by safety and device criteria. The current AMI processors are limited to $1,750 \mu \mathrm{A}$, but safety limits for the human array can be much lower depending on PD, such as $\sim 1,000 \mu \mathrm{A}$ for a PD of $205 \mu \mathrm{s}$ and $\sim 200 \mu \mathrm{A}$ for $984 \mu \mathrm{s}$. This is based on the safety equation for long-term central nervous system stimulation modeled by Shannon (1992) for a conservative condition $\left[\mathrm{ID}=\left(A \cdot 10^{k}\right)^{0.5}\right.$, where $k=1.5, I$ is the current threshold (in microamperes), $D$ is the phase duration (in seconds), and $A$ is site area (in squared centimeters)]. For more details on its derivation and application to activation levels in the first implanted AMI patients, see McCreery et al. (1988, 1990), Shannon (1992), and Lim et al. (2008b).
Figure 8 presents the evoked potential magnitudes as a function of stimulus level (in microamperes) for different PDs. The curves are separated into a caudal (blue circles) and rostral (green triangles) group. It is important to note that each plot does not consist of curves for all the locations listed in Table 1. For example, Figure $8 \mathrm{~A}$ is missing 3 caudal curves (only 9 of 12 shown) because the evoked potentials were not large enough with the levels we used for those cases to accurately measure the magnitude. However, a response was still present; thus, the threshold level could be identified for use in Figures 5 and 6. This occurred only for the caudal sites.

During the experiment, we attempted to stimulate each site to a level that elicited a reasonably large response of approximately $2-3 \mathrm{mV}$, which corresponds to the magnitude range observed for a broadband noise stimulus of about $60 \mathrm{~dB}$ SPL (Lim and Anderson 2007b). Some sites did not reach these magnitudes with our highest current level of $794 \mu \mathrm{A}$ (mostly caudal sites) or happened to be from one animal in which we did not stimulate to the highest levels. In general, as current level increases, the magnitudes increase with some curves exhibiting a saturating pattern. For a PD of $41 \mu \mathrm{s}$, the curves are spread out across the full current range with some overlap between rostral and caudal curves. However, the caudal curves dominate the right side of the plot in which high current levels still elicit small evoked potentials (the three most right curves are from the caudal region). There were also three additional caudal locations that did not elicit sufficient activation up to $794 \mu \mathrm{A}$ to obtain curves that would have been displayed even further to the right of the plot. As the PD increases, the rostral curves and a few of the caudal curves shift to the left reducing the differences observed among those curves. However, there are still a few caudal curves that remain spread out over a wide current range up to our maximum current limit of $794 \mu \mathrm{A}$ with a few caudal curve still not included in the plot due to insufficient activation levels (PD of 123 and $205 \mu \mathrm{s})$. This raises a potential issue for AMI implementation in which stimulation of some caudal regions will not elicit sufficient cortical activity even with extremely long PDs and high current levels that are beyond the safety limits for neural activation. This is further discussed in the Discussion: "Implications for an AMI".

Figure $8 \mathrm{G}$ provides additional data that more clearly demonstrate the difference in growth functions between rostral and caudal curves (for a PD of $205 \mu \mathrm{s})$. The data were collected from the eight animals implanted each with two simultaneous single shank arrays. Each animal had one or more pairs of sites in the same acoustically identified BF lamina in which one site was within a caudal region and the 
other site was in a more rostral region (1.5 mm apart). Neural responses in the same A1 location were recorded for each pair of site. This protocol reduced the data variability observed for the results presented in Figure 8A-F by ensuring comparison of a rostral region versus a caudal region in the same animal using the same A1 location with all sites within a similar BF region. The data in Figure 8A-F were pooled from different $\mathrm{BF}$ regions and $\mathrm{A} 1$ locations across animals that further required histological estimation (with additional errors) of the site locations within a rostral or caudal region. From Figure 8G, we clearly observe caudal curves that are shifted more to the right and with shallower slopes than the rostral curves. The slope of each curve was calculated by taking the difference in magnitude values divided by the difference in current values between the two endpoints of each curve, which assumes a linear slope. This method actually underestimates the initial portion of the curves (i.e., the steepest segment) for the rostral group compared to the caudal group. However, we still obtained significantly $(P \ll 0.001$, Student's paired $t$ test) steeper slopes for the rostral curves (mean 7.96, SD $4.6 \mu \mathrm{V} / \mu \mathrm{A}$ ) than for the caudal curves (mean 4.54 , SD $3.3 \mu \mathrm{V} / \mu \mathrm{A}$ ) even with this underestimation. These results suggest that evoked potentials are smaller for caudal stimulation versus rostral stimulation not only due to higher thresholds but also because of shallower growth functions. The curves did not span a large range of current levels as occurred for Figure 8A-F. This was a result of how we placed the two shanks into the ICC in which we intentionally, for other experimental purposes, inserted both shanks more rostrally to avoid the high threshold caudal-dorsal edge of the ICC.

\section{Latency versus location}

We observed a wide range of evoked potential latencies in A1 (8.1-18.1 ms) across different ICC stimulation locations and PDs. However, analyzing the contour plots for latencies versus ICC location, we did not identify any systematic trends with location or PD as we observed for thresholds and magnitudes. For this analysis, we did not plot the latencies across sites for a given current level above threshold. Instead, we plotted the peak latency corresponding to a specific peak magnitude within a set range (e.g., $0.6-0.9 \mathrm{mV}$ ) regardless of actual current level. From Figure 8, we observed that stimulation of more rostral-ventral regions generally exhibits steeper growth functions than stimulation of more caudal-dorsal regions. Thus, stimulation at a certain level above threshold could elicit a much larger evoked potential when stimulating a rostral-ventral location than a caudaldorsal location. If latencies decrease with increasing peak magnitude, then stimulation of rostral-ventral regions will appear to have shorter latencies simply due to larger evoked potentials that may mask latency differences directly caused by stimulation location. In general, evoked potential latencies decreased with increasing peak magnitude. Based on Table 1, there were a total of 132 different PD and location combinations (total sum of columns 2 and 3). We were able to plot the peak latency versus peak magnitude for 117 of these 132 cases. The remaining 15 cases did not have at least two points to extract the slope of the curve. The slope was calculated from the linear regression line through the points in which 109 out of 117 slope values were negative (mean -1.8 , SD $1.6 \mathrm{~ms} / \mathrm{mV}$ ) while only 8 were positive (mean 0.3 , SD $0.3 \mathrm{~ms} / \mathrm{mV}$ ). Thus, to avoid the confounding effects of latency shifts due to changes in evoked potential magnitudes, we plotted latencies across sites for a similar peak magnitude range instead of a set current level above threshold. This ensured that for a given contour plot, the latencies corresponded to evoked potentials with similar peak magnitudes across sites, and any systematic shifts in latency could be attributed to stimulation location across the isofrequency domain of the ICC.

We plotted the latency contour plots for specific PDs and peak magnitude ranges that we had a reasonable number of data points for statistical evaluation. Unlike the analyses for threshold, it was not possible to find a peak magnitude range that included all 21 stimulation locations. Two magnitude ranges provided at least 10 points for analysis for different PDs: 0.6-0.9 mV (PD of $82,123,205,328,656,984 \mu \mathrm{s}$ ) and $0.91-1.21 \mathrm{mV}$ (PD of 328 and $656 \mu \mathrm{s})$. Similar to the analyses performed for threshold and evoked potential magnitudes in Figures 6 and 7 , respectively, we identified the steepest gradient axis and performed a linear least-squares fit to the data points. None of the curves exhibited any significant trends in latency shifts with stimulation location as occurred with threshold and evoked potential magnitudes (see Table 2; all $P$ values considerably larger than 0.05 in contrast to the $P$ values obtained in Figs. 6 and 7 ). Even if the threshold or magnitude contour plots did not produce a statistically significant trend, we could still visually identify a general shift in values across the contour plots that were consistent across PDs and levels. This was not possible with the latency contour plots since they varied substantially across PD and peak magnitude ranges. We cannot rule out that inaccuracies in our electrophysiological and histological reconstruction methods may have masked any systematic location trends for latencies. However, the fact that a systematic organization of latency with ICC location was observed for a previous study using smaller stimulation sites (Lim and Anderson 2007a), it is possible that the considerably larger sites used in our study $\left(126,000 \mu \mathrm{m}^{2}\right.$ versus $400 \mu \mathrm{m}^{2}$ ) may have resulted in broader activation 
TABLE 2

Statistical summary for systematic latency shifts as a function of stimulation location along the isofrequency domain of the ICC

\begin{tabular}{|c|c|c|c|c|}
\hline Magnitude range $[\mathrm{mV}]$ & $P D[\mu s]$ & Number of locations & $R^{2}$ value & $P$ value \\
\hline $0.6-0.9$ & 82 & 10 & 0.03 & 0.65 \\
\hline $0.6-0.9$ & 123 & 11 & 0.09 & 0.37 \\
\hline $0.6-0.9$ & 205 & 10 & 0.16 & 0.25 \\
\hline $0.6-0.9$ & 328 & 14 & 0.01 & 0.72 \\
\hline $0.6-0.9$ & 656 & 10 & 0.04 & 0.56 \\
\hline $0.6-0.9$ & 984 & 12 & 0.06 & 0.45 \\
\hline $0.9-1.21$ & 328 & 11 & 0.00 & 0.87 \\
\hline $0.91-1.21$ & 656 & 10 & 0.23 & 0.23 \\
\hline
\end{tabular}

Similar to the analyses performed for threshold and evoked potential magnitudes in Figures 6 and 7 , respectively, we identified the steepest gradient axis from the latency contour plots and performed a linear least-squares fit to the data points along this steepest gradient axis. The $P$ and $R^{2}$ values are listed above for the different peak magnitude ranges and phase durations (PDs) used for analysis that consisted of at least ten location points. None of the cases produced any significant trends $(P \gg 0.05)$ for latency shifts as a function of stimulation location. For more details on the analysis method, see Results: "Latency versus location"

patterns and led to more complex changes with PD and activation levels.

\section{DISCUSSION}

In summary, we observed that electrical stimulation along the isofrequency domain of the ICC exhibits systematic changes in evoked potential thresholds and magnitudes recorded in the main input layer of A1. In particular, stimulation of more rostral-ventral regions requires lower stimulation thresholds, elicits greater evoked activity, and generally exhibits steeper evoked potential growth functions. This trend is the clearest at lower PDs (at least down to the lowest PD we tested of $41 \mu \mathrm{s})$ and becomes less significant with longer PDs. Furthermore, stimulation of caudal-dorsal regions resulted in much greater variability in thresholds and magnitude growth functions across PDs than stimulation of more rostral-ventral regions. Overall, our results demonstrate that there are clear differences in activation properties along the isofrequency domain of the ICC to electrical stimulation with our human AMI array, consistent with a previous study ( $\mathrm{Lim}$ and Anderson 2007a) that used considerably smaller sites (400 $\mu^{2}$ versus $126,000 \mu \mathrm{m}^{2}$ ).

\section{Possible mechanisms for the caudal-rostral effects}

It is still not clear as to what may be causing the activation differences between the caudal-dorsal and rostral-ventral regions along the isofrequency domain of the ICC. As discussed in Lim et al. (2008a), there is anatomical evidence that different distributions of brainstem nuclei project differentially to the caudal-medial region (i.e., caudal-dorsal region along the isofrequency domain in our coordinate system) versus the rostral-lateral region (i.e., rostral-ventral region) in gerbils (Cant and Benson 2006). These regions also maintain some segregation to the ventral division of the medial geniculate nucleus (MGv) in which the caudal-medial ICC region projects to the caudal one-third of the $\mathrm{MGv}$ and the rostral-lateral ICC region projects to the rostral two-thirds of the MGv (Cant and Benson 2007). Interestingly, a caudal-rostral organization within the MGv has also been shown in cats, in which A1 (and the anterior auditory field) receives most of its projections from the rostral MGv but few from the caudal MGv (it projects more to the posterior auditory field) (Niimi and Matsuoka 1979; Morel and Imig 1987; Rodrigues-Dagaeff et al. 1989). It was further shown by Rodrigues-Dagaeff et al. (1989) that the caudal MGv generally exhibits longer and more widely distributed latencies, a less defined tonotopic organization, less excitatory responses, degraded synchronization properties, and systematic differences in other sound features compared to the rostral MGv. This is interesting in that, traditionally, the ICC has been viewed as a single nucleus with properties associated with the lemniscal pathway (i.e., good tonotopy and projections to the MGv and A1), which does not appear to be accurate based on these findings in gerbils and cats. Based on these findings, it is possible that neurons activated within the caudal ICC project predominantly to other cortical regions than $\mathrm{A} 1$, thus requiring greater stimulation levels for current to spread and activate more rostral neurons (or other surrounding neurons) that then project to A1. This could explain the extremely high current levels required for caudal ICC stimulation to effectively elicit activity in A1.

Another possibility for the caudal-rostral differences is that the caudal pathway exhibits less excitability and requires greater current levels to achieve effective activation of A1. The reduced excitability can be associated with complex neural interactions (i.e., requiring accurate timing of activation across neurons) or inhibitory mechanisms within the IC (Le Beau et al. 1996; LeBeau et al. 2001; Kelly and Caspary 2005; Wu 
2005) and/or within the medial geniculate nucleus (Winer et al. 1996; Peruzzi et al. 1997; Bartlett et al. 2000; Ito et al. 2009; Lee and Sherman 2010) that may not sufficiently respond to the artificially synchronized activation of groups of neurons induced by electrical stimulation. There is some evidence in the cat that greater inhibitory patterns are observed in caudal ICC regions compared to more rostral regions in response to ipsilateral pure tone stimulation based on 2-deoxyglucose mapping (Webster et al. 1984). However, no other study to our knowledge has demonstrated a general dominance of inhibitory circuits within the caudal ICC that would strongly suggest that the differential stimulation effects are attributed to neural activation properties within the ICC. Furthermore, electrical stimulation of the ICC will activate output projections to the thalamus that are distributed throughout the ICC (Coomes and Schofield 2004; Cant and Benson 2007). Presynaptic axons and interneurons will also be activated by ICC stimulation. However, their spikes will generally be blocked by the simultaneously activated postsynaptic neurons projecting to the thalamus that are temporarily in a refractory state (McIntyre and Grill 1999; McIntyre et al. 2004). Even if there was a dominant inhibitory input into the caudal ICC that could be electrically activated and suppress output neurons to the thalamus, there would still be other nearby output neurons that could be simultaneously activated to then transmit spikes to the thalamus. Thus, it seems more likely that the observed caudalrostral differences for ICG stimulation are associated with output projections to the medial geniculate nucleus rather than the input circuitry within the ICC.

Animal studies have demonstrated that projections from the IC to the medial geniculate nucleus are both excitatory and inhibitory (Winer et al. 1996; Peruzzi et al. 1997; Bartlett et al. 2000). Furthermore, the largest tectothalamic neurons are inhibitory (i.e., GABAergic), represent up to $45 \%$ (in rat; $20 \%$ in cat) of the ascending projections, and provide fast transmission to the medial geniculate nucleus that can precede the excitatory tectothalamic input (Winer et al. 1996; Peruzzi et al. 1997; Ito et al. 2009). Although these inhibitory projections do not appear to be clustered only within the caudal ICC, it is possible that a specific inhibitory circuitry within the medial geniculate nucleus suppresses thalamic activity to electrical stimulation of the caudal ICC. The excitatory nature of projections from the medial geniculate nucleus to the main input layers of A1 (i.e., III/IV) (Cruikshank et al. 2002; Broicher et al. 2010) further suggests that the suppressive mechanism to caudal ICC stimulation may occur within the thalamus. However, as discussed above, the lack of A1 activity could also be attributed to a lack of projections from the caudal ICC to A1 via the thalamus (i.e., caudal ICC projects to caudal MGv that does not predominantly project to A1).

\section{Implications for an AMI}

Using single biphasic pulses in our study, stimulation of the caudal-dorsal ICC region required high current levels for activation. We obtained current thresholds exceeding our maximum stimulator level of $794 \mu \mathrm{A}$ (equal to $58 \mathrm{~dB}$; Figs. 6 and 8). This is consistent with a previous study that stimulated the ICG with $400 \mu \mathrm{m}^{2}$ sites with a PD of $200 \mu$ s (Lim and Anderson 2007a). They obtained thresholds exceeding their maximum stimulator level of $60 \mu \mathrm{A}$ for caudal stimulation but thresholds lower than $10 \mu \mathrm{A}$ for rostral stimulation. In our study, caudal thresholds could be as high as $400 \mu \mathrm{A}(48 \mathrm{~dB})$ while rostral thresholds could be as low as about $50 \mu \mathrm{A}(24 \mathrm{~dB})$ for a PD of $205 \mu \mathrm{s}$. Thresholds were much higher for stimulation with our AMI sites, which may be attributed to the considerably larger sites. Nevertheless, it is surprising that the thresholds obtained in both studies are extremely high considering that the sites are in direct contact with the target neural elements. A cochlear implant (CI) study using a similar guinea pig model obtained a median threshold of $70 \mu \mathrm{A}$ (minimum $24 \mu \mathrm{A}$, maximum $134 \mu \mathrm{A}$ ) for A1 spike thresholds in response to stimulation of a six-electrode Nucleus array (Cochlear Ltd., Englewood, CO, USA) implanted into the cochlea (Bierer and Middlebrooks 2002). These threshold ranges are consistent with other CI studies in cats (Raggio and Schreiner 1994, 1999). CI stimulation must transmit current through a bony modiolar wall to activate distant auditory nerve fibers yet still exhibit significantly lower thresholds than required for effective stimulation of the caudal ICC.

We were able to reduce overall current levels for activation by increasing PD in which all rostral curves and most of the caudal curves shifted to the left in Figure 8. However, increasing PD results in greater overall energy requirements (Fig. 5C), and unfortunately, there are still a few caudal regions in which stimulation at extremely high current levels and long PDs beyond safety limits still could not elicit reasonably large evoked potentials $(\sim 2-3 \mathrm{mV}$ corresponding to the magnitudes induced by a $\sim 60$-dB SPL noise burst). For example, at a PD of $984 \mu$ s, the safety limit is about $200 \mu \mathrm{A}$ (Shannon 1992), which is far lower than needed to increase the evoked potentials to a reasonable magnitude for the three most right caudal curves in Figure 8F. It is possible that more complex stimuli (e.g., appropriate inter-stimulus delays across sites along the isofrequency domain) may avoid inhibitory effects caused by artificial activation of the caudal ICC and more effectively activate A1 than single pulse stimulation. However, at this stage, it is 
not clear how this can be achieved. Furthermore, this is assuming that there are projections from the caudal ICC to A1 via the medial geniculate nucleus.

An alternative solution is to simply position the AMI array into a rostral-ventral region in future patients to ensure low threshold activation. This would also enable the use of a short PD (e.g., $123 \mu$ s that provides an optimal balance between energy and level requirements; Fig. 5), which is necessary for the implant stimulation strategy when attempting to stimulate with faster pulse rates across sites. However, the question arises as to what information will be lost by stimulating only the rostral-ventral region of the ICC. Projections originating from different brainstem nuclei terminate into overlapping and non-overlapping regions throughout the ICC (Roth et al. 1978; BrunsoBechtold et al. 1981; Ramachandran and May 2002; Loftus et al. 2004; Cant and Benson 2006). Considering the distinct anatomical and functional properties across neurons within the different brainstem nuclei that project to the ICC (Cant and Benson 2003; Cant 2005; Schofield 2005), especially the neurons within the cochlear nucleus important for speech coding (Blackburn and Sachs 1990; Winter and Palmer 1990; Recio and Rhode 2000), it is expected that different and important features of sound are being transmitted to specific areas of the ICC. There is some evidence of a caudal-rostral organization of brainstem inputs into the ICC. Loftus et al. (2004) observed in cats that within lower frequency laminae (less than $5 \mathrm{kHz}$ ) projections from the medial superior olivary nucleus were more frequently located in caudal ICC regions while those from the lateral superior olivary nucleus dominated in more rostral regions. For higher frequency laminae, it appeared that inputs from the dorsal cochlear nucleus projected throughout the laminae but inputs from the lateral superior olivary dominated more ventrallateral regions. These lateral superior olivary inputs also appeared to be further segregated in terms of their contralateral and ipsilateral origins. Cant and Benson (2006) demonstrated in gerbils that the cochlear nucleus and the nuclei of the lateral lemniscus project throughout the ICC but that the nuclei of the superior olivary complex project predominantly to the rostral-lateral regions. Although there appears to be some differences across species in the spatial organization of brainstem projections within the ICC, it is apparent that stimulation of a specific ICC region, such as the rostral-ventral region, will transmit only a limited portion of sound information to higher auditory centers. However, within this limited representation, the question is whether or not there is sufficient information to at least restore basic features important for speech perception in a quiet environment, which would still be a major success for the AMI.
The phenomenal success of CIs has been largely attributed to the brain's ability to extract essential features of speech from crude electrical activation patterns. CI-based strategies generally filter the incoming sound into different bandpass signals (i.e., frequency channels) that then extract the envelope of those signals to amplitude modulate electrical pulse trains transmitted to each site located in a specific best frequency region of the cochlea. At least in quiet environments, humans can still achieve intelligible speech perception even with crude temporal $(<50 \mathrm{~Hz}$ modulations) and spectral ( $<4$ bands) cues (Shannon et al. 1995, 2004). As long as there is a sufficient representation of the slow modulations of the sound input and a few channels of frequency information, implant patients should be able to obtain reasonable speech perception in quiet. This has been demonstrated in ABI patients, particularly those without tumors, who have achieved high levels of speech perception (i.e., comparable to some of the top CI patients) with CIbased strategies (Colletti and Shannon 2005; Colletti et al. 2009) even though they are receiving crude temporal and spatial activation patterns predominantly across the dorsal cochlear nucleus, which is not the main ascending nuclei for speech features (Blackburn and Sachs 1990; Recio and Rhode 2000; Cant and Benson 2003). Similar success should be possible with AMI stimulation assuming that sufficient temporal and spectral information can be transmitted to higher auditory centers.

There is some evidence that stimulation of the rostral-ventral ICC region with CI-based strategies may be successful. ICC neurons still appear to exhibit some general representation of the stimulus waveform as occurs at the cochlear level (Cariani and Delgutte 1996; Suta et al. 2003). In other words, many neurons still respond in synchrony to the envelope of the sound signal and with a spatial representation for frequency information (Krishna and Semple 2000; Joris et al. 2004). This neural representation of the stimulus becomes more complex and abstract in higher auditory centers as neurons begin to create an internal representation for the different features of the stimulus rather than a direct representation of the stimulus waveform (Wang et al. 2008). However, as long as ICC neurons can still sufficiently phase-lock to the slow temporal features required for speech perception, then the amplitude-modulated pulse sequences of CI-based strategies may prove effective. Neurons within the rostral-ventral region along the isofrequency domain of the ICC have shown to exhibit shorter latencies and higher best modulation frequencies (i.e., can follow and represent faster amplitude-modulated stimuli) than those in more caudal-dorsal regions (Langner et al. 1987, 2002; Schreiner and Langner 1988). There may exist some 
variation in this periodotopic representation across different sound levels (Krishna and Semple 2000). Nevertheless, this general finding is consistent with our results as well as those described in the previous section in that the rostral ICC pathway may represent a more robust channel for transmitting temporal (as well as level and spectral) features to higher auditory centers compared to the caudal pathway.

Based on the discussion above, rostral-ventral ICC stimulation with a single shank array may be sufficient to restore open-set speech perception in a quiet environment. Consistent with our animal findings, the one AMI patient currently implanted into a caudal-dorsal ICC region has high perceptual thresholds $(6-12 \mathrm{nC})$ (Lim et al. 2007), which is comparable to CI thresholds (5$20 \mathrm{nC}$ using approximately similar stimuli; Shannon 1985; Pfingst et al. 1997). This consistency raises the possibility that the limited hearing performance in the AMI patient is due to the poor temporal, spectral, and level coding properties associated with the caudaldorsal ICG identified across animal studies. Even though speech perception in quiet can be achieved with slow modulation information that could be transmitted by caudal-dorsal ICC neurons, the need and consequences for extremely high current levels for activation may be responsible for the limited hearing performance. At least the rostral-ventral ICC pathway can be effectively activated with low current levels and may be better suited for AMI implantation in future patients. However, rostral-ventral ICC neurons appear to code for modulation frequencies much higher than required for speech perception $(>100 \mathrm{~Hz})$ though some of these neurons can still code for lower modulation frequencies (Krishna and Semple 2000; Langner et al. 2002). Thus, the question remains if artificial electrical stimulation of rostral-ventral neurons can still provide sufficient modulation cues for lower frequencies with the added advantage of being able to also transmit higher frequency information. The central activation patterns induced by ICC stimulation do not necessarily have to match what normally occurs to acoustic stimulation, but just need to provide sufficient modulation cues to understand basic speech elements.

Another consideration is that the ICC is a complex nucleus that normally codes information across a three-dimensional structure. Therefore, we may still need to implant a three-dimensional array to sufficiently activate different regions throughout the ICC in appropriate temporal and level patterns to restore open-set speech perception. Stimulating just the rostral-ventral ICC regions may not be sufficient. Thus, we are currently investigating in animals the benefit of multi-site stimulation as well as possible three-dimensional array designs that could be safely implanted into humans.

\section{ACKNOWLEDGMENTS}

We would like to thank Verena Scheper for assistance with animal surgeries and Cornelia Froemke (Center of Biometry, Medical Informatics and Medical Technology, Hannover Medical University) for assistance with data analysis. We would also like to thank James Patrick and Frank Risi (Cochlear Ltd., Lane Cove, Australia) for technical support with and development of the AMI array. This research was funded by Cochlear Ltd. and German Research Foundation (SFB 599).

\section{REFERENCES}

Abeles M, Goldstein MH JR (1970) Functional architecture in cat primary auditory cortex: columnar organization and organization according to depth. J Neurophysiol 33:172-187

Bartlett EL, Stark JM, Guillery RW, Smith PH (2000) Comparison of the fine structure of cortical and collicular terminals in the rat medial geniculate body. Neuroscience 100:811-828

Bierer JA, Middlebrooks JC (2002) Auditory cortical images of cochlear-implant stimuli: dependence on electrode configuration. J Neurophysiol 87:478-492

Blackburn CC, Sachs MB (1990) The representations of the steadystate vowel sound /e/ in the discharge patterns of cat anteroventral cochlear nucleus neurons. J Neurophysiol 63:1191-1212

Bledsoe SC, Shore SE, Guitton MJ (2003) Spatial representation of corticofugal input in the inferior colliculus: a multicontact silicon probe approach. Exp Brain Res 153:530-542

Brackmann DE, Hitselberger WE, Nelson RA, Moore J, Waring MD, Portillo F, Shannon RV, Telischi FF (1993) Auditory brainstem implant: I Issues in surgical implantation. Otolaryngol Head Neck Surg 108:624-633

Broicher T, Bidmon HJ, Kamuf B, Coulon P, Gorji A, Pape HC, Speckmann EJ, Budde T (2010) Thalamic afferent activation of supragranular layers in auditory cortex in vitro: a voltage sensitive dye study. Neuroscience 165:371-385

Brunso-Bechtold JK, Thompson GC, Masterton RB (1981) HRP study of the organization of auditory afferents ascending to central nucleus of inferior colliculus in cat. J Comp Neurol 197:705-722

CAnT NB (2005) Projections from the cochlear nuclear complex to the inferior colliculus. In: Winer JA, Schreiner CE (eds) The inferior colliculus. Springer Science+Business Media, New York, pp 115-131

Cant NB, Benson CG (2003) Parallel auditory pathways: projection patterns of the different neuronal populations in the dorsal and ventral cochlear nuclei. Brain Res Bull 60:457-474

Cant NB, Benson CG (2006) Organization of the inferior colliculus of the gerbil (Meriones unguiculatus): differences in distribution of projections from the cochlear nuclei and the superior olivary complex. J Comp Neurol 495:511-528

Cant NB, Benson CG (2007) Multiple topographically organized projections connect the central nucleus of the inferior colliculus to the ventral division of the medial geniculate nucleus in the gerbil, Meriones unguiculatus. J Comp Neurol 503:432-453

Cariani PA, Delgutte B (1996) Neural correlates of the pitch of complex tones. I. Pitch and pitch salience. J Neurophysiol 76:1698-1716

Colletti V, Shannon RV (2005) Open set speech perception with auditory brainstem implant? Laryngoscope 115:1974-1978

Colletti V, Shannon R, Carner M, Veronese S, Colletti L (2009) Outcomes in nontumor adults fitted with the auditory brainstem implant: 10 years' experience. Otol Neurotol 30:614-618 
Coomes DL, Schofield BR (2004) Separate projections from the inferior colliculus to the cochlear nucleus and thalamus in guinea pigs. Hear Res 191:67-78

Cruikshank SJ, Rose HJ, Metherate R (2002) Auditory thalamocortical synaptic transmission in vitro. J Neurophysiol 87:361-384

DiCarlo JJ, Lane JW, Hsiao SS, Johnson KO (1996) Marking microelectrode penetrations with fluorescent dyes. J Neurosci Methods 64:75-81

Friesen LM, Shannon RV, Baskent D, Wang X (2001) Speech recognition in noise as a function of the number of spectral channels: comparison of acoustic hearing and cochlear implants. J Acoust Soc Am 110:1150-1163

HuAng CL, Winer JA (2000) Auditory thalamocortical projections in the cat: laminar and areal patterns of input. J Comp Neurol 427:302-331

Ito T, Bishop DC, Oliver DL (2009) Two classes of GABAergic neurons in the inferior colliculus. J Neurosci 29:13860-13869

Joris PX, Schreiner CE, ReEs A (2004) Neural processing of amplitude-modulated sounds. Physiol Rev 84:541-577

Keliy JB, Caspary DM (2005) Pharmacology of the inferior colliculus. In: Winer JA, Schreiner CE (eds) The inferior colliculus. Springer Science+Business Media, New York, pp 248-281

Kral A, Hartmann R, Tillein J, Heid S, Klinke R (2000) Congenital auditory deprivation reduces synaptic activity within the auditory cortex in a layer-specific manner. Cereb Cortex 10:714-726

Krishna BS, Semple MN (2000) Auditory temporal processing: responses to sinusoidally amplitude-modulated tones in the inferior colliculus. J Neurophysiol 84:255-273

Langner G, Schreiner C, Merzenich MM (1987) Covariation of latency and temporal resolution in the inferior colliculus of the cat. Hear Res 31:197-201

LANGner G, Albert M, Briede T (2002) Temporal and spatial coding of periodicity information in the inferior colliculus of awake chinchilla (Chinchilla laniger). Hear Res 168:110-130

Le Beau FE, Rees A, Malmierca MS (1996) Contribution of GaBAand glycine-mediated inhibition to the monaural temporal response properties of neurons in the inferior colliculus. J Neurophysiol 75:902-919

LeBeau FE, Malmierca MS, Rees A (2001) Iontophoresis in vivo demonstrates a key role for GABA(A) and glycinergic inhibition in shaping frequency response areas in the inferior colliculus of guinea pig. J Neurosci 21:7303-7312

Lee CC, Sherman SM (2010) Topography and physiology of ascending streams in the auditory tectothalamic pathway. Proc Natl Acad Sci USA 107:372-377

Lenarz M, Lim HH, Patrick JF, Anderson DJ, Lenarz T (2006a) Electrophysiological validation of a human prototype auditory midbrain implant in a guinea pig model. J Assoc Res Otolaryngol 7:383-398

Lenarz T, Lim HH, Reuter G, Patrick JF, Lenarz M (2006в) The auditory midbrain implant: a new auditory prosthesis for neural deafnessconcept and device description. Otol Neurotol 27:838-843

Lim HH, Anderson DJ (2006) Auditory cortical responses to electrical stimulation of the inferior colliculus: implications for an auditory midbrain implant. J Neurophysiol 96:975-988

Lim HH, ANDERSON DJ (2007A) Spatially distinct functional output regions within the central nucleus of the inferior colliculus: implications for an auditory midbrain implant. J Neurosci 27:8733-8743

Lim HH, Anderson DJ (2007в) Antidromic activation reveals tonotopically organized projections from primary auditory cortex to the central nucleus of the inferior colliculus in guinea pig. J Neurophysiol 97:1413-1427

Lim HH, Lenarz T, Joseph G, Battmer RD, Samil A, Samit M, Patrick JF, LeNarz M (2007) Electrical stimulation of the midbrain for hearing restoration: insight into the functional organization of the human central auditory system. J Neurosci 27:1354113551

Lim HH, Lenarz T, Anderson DJ, Lenarz M (2008A) The auditory midbrain implant: effects of electrode location. Hear Res 242:74-85

Lim HH, Lenarz T, Joseph G, Battmer RD, Patrick JF, Lenarz M (2008B) Effects of phase duration and pulse rate on loudness and pitch percepts in the first auditory midbrain implant patients: comparison to cochlear implant and auditory brainstem implant results. Neuroscience 154:370-380

Lim HH, Lenarz M, Lenarz T (2009) Auditory midbrain implant: a review. Trends Amplif 13:149-180

Loftus WC, Bishop DC, Saint Marie RL, Oliver DL (2004) Organization of binaural excitatory and inhibitory inputs to the inferior colliculus from the superior olive. J Comp Neurol 472:330-344

Loizou PC, Dorman M, Fitzke J (2000) The effect of reduced dynamic range on speech understanding: implications for patients with cochlear implants. Ear Hear 21:25-31

Malmierca MS, Rees A, Le Beau FE, Bjaalie JG (1995) Laminar organization of frequency-defined local axons within and between the inferior colliculi of the guinea pig. J Comp Neurol 357:124-144

McCreery DB, Agnew WF, Yuen TG, Bullara LA (1988) Comparison of neural damage induced by electrical stimulation with faradaic and capacitor electrodes. Ann Biomed Eng 16:463-481

McCreery DB, Agnew WF, Yuen TG, Bullara L (1990) Charge density and charge per phase as cofactors in neural injury induced by electrical stimulation. IEEE Trans Biomed Eng 37:996-1001

MCINTyRe CC, Grill WM (1999) Excitation of central nervous system neurons by nonuniform electric fields. Biophys J 76:878-888

McIntyre CC, Grill WM, Sherman DL, Thakor NV (2004) Cellular effects of deep brain stimulation: model-based analysis of activation and inhibition. J Neurophysiol 91:1457-1469

Merzenich MM, ReID MD (1974) Representation of the cochlea within the inferior colliculus of the cat. Brain Res 77:397-415

Middlebrooks JC (2008) Auditory cortex phase locking to amplitude-modulated cochlear implant pulse trains. J Neurophysiol 100:76-91

Mitzdorf U (1985) Current source-density method and application in cat cerebral cortex: investigation of evoked potentials and EEG phenomena. Physiol Rev 65:37-100

Morel A, Imig TJ (1987) Thalamic projections to fields A, AI, P, and VP in the cat auditory cortex. J Comp Neurol 265:119-144

Muller-Preuss P, Mitzdorf U (1984) Functional anatomy of the inferior colliculus and the auditory cortex: current source density analyses of click-evoked potentials. Hear Res 16:133-142

Nimi K, Matsuoka H (1979) Thalamocortical organization of the auditory system in the cat studied by retrograde axonal transport of horseradish peroxidase. Adv Anat Embryol Cell Biol 57:1-56

Peruzzi D, Bartlett E, Smith PH, Oliver DL (1997) A monosynaptic GABAergic input from the inferior colliculus to the medial geniculate body in rat. J Neurosci 17:3766-3777

Pfingst BE, Zwolan TA, Holloway LA (1997) Effects of stimulus configuration on psychophysical operating levels and on speech recognition with cochlear implants. Hear Res 112:247-260

RAGGio MW, SCHREINER CE (1994) Neuronal responses in cat primary auditory cortex to electrical cochlear stimulation. I. Intensity dependence of firing rate and response latency. J Neurophysiol 72:2334-2359

RAgGio MW, Schreiner CE (1999) Neuronal responses in cat primary auditory cortex to electrical cochlear stimulation. III. Activation patterns in short- and long-term deafness. J Neurophysiol 82:3506-3526

RamaChANDRAN R, MAY BJ (2002) Functional segregation of ITD sensitivity in the inferior colliculus of decerebrate cats. J Neurophysiol 88:2251-2261 
Rance G, Cone-Wesson B, Wunderlich J, Dowell R (2002) Speech perception and cortical event related potentials in children with auditory neuropathy. Ear Hear 23:239-253

Recio A, Rhode WS (2000) Representation of vowel stimuli in the ventral cochlear nucleus of the chinchilla. Hear Res 146:167184

Redies H, Sieben U, Creutzfeldt OD (1989) Functional subdivisions in the auditory cortex of the guinea pig. J Comp Neurol 282:473-488

Reuter G, Reich U, Marquardt N, Kuingberg M, Lenarz M, Lenarz T (2004) Frequency-specific activity of the auditory pathway with auditory midbrain implants. Biomed Tech 49:896-897

Rodrigues-Dagaeff C, Simm G, De Ribaupierre Y, Villa A, De Ribaupierre F, Roulller EM (1989) Functional organization of the ventral division of the medial geniculate body of the cat: evidence for a rostro-caudal gradient of response properties and cortical projections. Hear Res 39:103-125

Roth GL, Aitkin LM, Andersen RA, Merzenich MM (1978) Some features of the spatial organization of the central nucleus of the inferior colliculus of the cat. J Comp Neurol 182:661-680

Samil A, Lenarz M, Majdani O, Lim HH, Sami M, Lenarz T (2007) Auditory midbrain implant: a combined approach for vestibular schwannoma surgery and device implantation. Otol Neurotol 28:31-38

SCHOFIELD BR (2005) Superior olivary complex and lateral lemniscal connections of the auditory midbrain. In: Winer JA, Schreiner CE (eds) The inferior colliculus. Springer Science+Business Media, New York, pp 132-154

Schreiner CE, Langner G (1988) Periodicity coding in the inferior colliculus of the cat. II. Topographical organization. J Neurophysiol 60:1823-1840

Schreiner CE, Langner G (1997) Laminar fine structure of frequency organization in auditory midbrain. Nature 388:383386

Schwartz MS, Otto SR, Shannon RV, Hitselberger WE, Brackmann DE (2008) Auditory brainstem implants. Neurotherapeutics 5:128-136

SHANNON RV (1985) Threshold and loudness functions for pulsatile stimulation of cochlear implants. Hear Res 18:135-143
Shannon RV (1992) A model of safe levels for electrical stimulation. IEEE Trans Biomed Eng 39:424-426

Shannon RV, Zeng FG, Kamath V, Wygonski J, Ekelid M (1995) Speech recognition with primarily temporal cues. Science 270:303-304

Shannon RV, Fu QJ, GALvin J 3RD (2004) The number of spectral channels required for speech recognition depends on the difficulty of the listening situation. Acta Otolaryngol 124 (Suppl):50-54

Sмith PH, Populin LC (2001) Fundamental differences between the thalamocortical recipient layers of the cat auditory and visual cortices. J Comp Neurol 436:508-519

SNyder RL, Bierer JA, Middlebrooks JC (2004) Topographic spread of inferior colliculus activation in response to acoustic and intracochlear electric stimulation. J Assoc Res Otolaryngol 5:305-322

Suta D, Kvasnak E, Popelar J, Syka J (2003) Representation of species-specific vocalizations in the inferior colliculus of the guinea pig. J Neurophysiol 90:3794-3808

Wallace MN, Rutkowski RG, Palmer AR (2000) Identification and localisation of auditory areas in guinea pig cortex. Exp Brain Res 132:445-456

WANG X, Lu T, Bendor D, Bartlett E (2008) Neural coding of temporal information in auditory thalamus and cortex. Neuroscience 154:294-303

Webster WR, SERVIERE J, BRown M (1984) Inhibitory contours in the inferior colliculus as revealed by the 2-deoxyglucose method. Exp Brain Res 56:577-581

Winer JA, Saint Marie RL, Larue DT, Oliver DL (1996) GaBAergic feedforward projections from the inferior colliculus to the medial geniculate body. Proc Natl Acad Sci USA 93:8005-8010

Winter IM, Palmer AR (1990) Temporal responses of primarylike anteroventral cochlear nucleus units to the steady-state vowel $/ \mathrm{i}$. J Acoust Soc Am 88:1437-1441

WU SH (2005) Biophysical properties of inferior colliculus neurons. In: Winer JA, Schreiner CE (eds) The inferior colliculus. Springer Science+Business Media, New York, pp 282-311

Zeng FG, GaLvin JJ 3RD (1999) Amplitude mapping and phoneme recognition in cochlear implant listeners. Ear Hear 20:60-74 